\title{
ENRICO CELANI
}

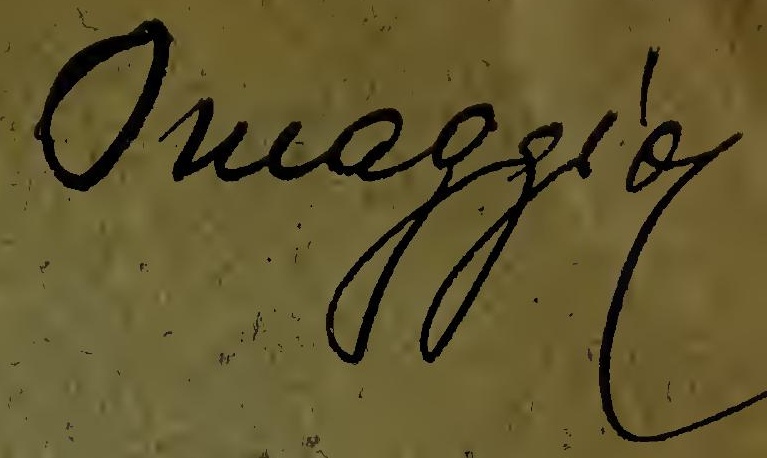

Soppa un Epbario di Ghepardo Cibo

\author{
consepvato
}

nella R. Biblioteca Angelica di Roma

GENOVA

TIPOGRAFIA DI ANGELO CIMINAGO

Vico Mele 7, int. 5 e 6 .

1902: 


\title{
ENRICO CELANI
}

\section{Soppa un Epbapio di Ghepapdo Cibo}

\author{
consepvato
}

nella R. Bibliotesa Angelipa di Roma

\author{
GENOVA \\ TIPOGRAFIA DI ANGELO CIMINAGO \\ Vico Mele 7 , int. 5 e 6 \\ 1902.
}


92008 
Nella sala dei manoscritti della R. Biblioteca Angelica di Roma, giacevano da inolti anni, ficcati in uno scaffale, senza alcuna collocazione o registrazione in catalogo, cinque volumi di erbario: polverosi, mal ridotti, rovinati nelle legature e nelle coperture tanto da esigere, specialmente uno di essi, notevoli e radicali riparazioni. L'attuale bibliotecario, prof. Gennaro Buonanno, provvide affinchè fossero restaurati, ed oggi, conservati in cassette chiuse e foderate di ovatta spalmata di nafta, sono una delle non poche preziosità che conserva quella biblioteca.

A rilevare l'importanza di questo ignoto erbario Angelicano furono i primi i professori Pirotta e Chiovenda, i quali, appena ne conobbero l' esistenza, recatisi all'Angelica, lo esaminarono e con quella competenza a loro universalmente riconosciuta, lo giudicarono composto nella seconda metà del secolo XVI. E il loro giudizio non andò molto lungi dal vero. Il prof. Chiovenda poi, basandosi sull'esame intrinseco di esso, tentò rinvenirne l' autore; ma dopo alquanto tempo dichiarò che le sue fatiche erano rimaste senza risultato, perchè l'erbario non offriva alcun indizio che potesse, anche lontanamente, dare luogo a ricerche. Volli tentare ancor io la prova: e dacchè la grande competenza del prof. Chiovenda escludeva che dall'esame di esso, dall'assieme delle sue piante e dalla loro nomenclatura potesse venirne fuori una traccia, mi proposi battere tutt' altra via, abbandonando completamente quella dell' erbario, e sono lieto di essere riuscito oltre le mie speranze.

Benché se ne ignorasse" completamente l' autore, era già molto potere accertare in cquale epoca esso fosse pervenuto alla biblioteca, e per questo eranvi dati precisi. 
In un opuscolo stampato l'anno $1608\left({ }^{1}\right)$ che può dirsi una specie d' indice per classi della Biblioteca, a pagina 57 leggesi sotto il titolo: RES ARBOREA ET HERBARIA: Herbae, ac plantae reapse super chartam conglutinatae pluribus tomis in folio comprehensae, ed evidentemente questo si riferisce al nostro erbario. Dunque l'anno 1608, epoca in cui fu stampato quell' opuscolo, l' erbario era nell'Angelica: e forse non è azzardato l'asserire che, provenendo esso dal P. Angelo Rocca $\left({ }^{2}\right)$ come tutto il primo fondo che compose la biblioteca, esso vi stesse già dall'anno 1605, anno in cui il Rocca dispose che i suoi libri e manoscritti fossero affidati all'Ordine Agostiniano e-in servizio degli studiosi. Ma nulla più di questa notizia: gli indici e cataloghi posteriori tacevano assolutamente dell'erbario: di modo che poteva asserirsi che per un periodo di tre secoli nessuno dei tanti bibliotecarii dell'Angelica ne aveva compresa l'importanza: anzi considerandolo come un ingombro non avevano perduto tempo neppure a catalogarlo.

Esaminando l'indice delle piante contenute nell'erbario, un piccolo manoscritto oblungo del quale tratterò in seguito, e notando che vicino ai nomi di esse piante, l'anonimo erborizzatore avera spesso registrato nomi di autori di opere botaniche, quali Plinio, Fuchs, Mattioli, Teofrasto, Rucllio, Manardo, pensai che egli, oltre il conoscere quegli autori, ne dovesse possedere le opere per citare con tanta franchezza e precisione: e conseguentemente mi chiesi se insieme all' erbario, non fossero pervenuti al-

(1) Bibliotheca Angelica litteratorum litterarumq. amatorum commoditati dicata Romae in Aedibus Augustinianis. Romae, St. Paulinum, MICVIII.

(2) Angelo Rocca, nato nel 1545 a Rocca Contrada nelle Marche (l'attuale Arcevia), all'età di sette anni vesti l'abito degli Eremitani di S. Agostino nel convento di Camerino: dipoi venne, Maestro in sacra teologia, nel convento generalizio di Roma. Nel 1585 fu chiamato da Sisto V a sopraintendere alla Stamperia Vaticana: nel 1595 divenne Prefetto del Sacrario Apostolico, e nel 1605 vescovo di Tagaste in partibus. Mori a Roma nol 1620. Possedeva una ricea biblioteca che nel 1605 affidó agli Agostiniani, confermando poi questa sua disposizione con istromento del 23 ottobre 1614 in atti Celso Cusano N. C: 
l'Angelica anche i libri che lo averano servito ed aiutato, e'se da essi - dato che vi fossero - non potessi trarre qualche nota, un qualunque indizio che mi aprisse la via alla ricerca, non essendo difficile nè raro trovare edizioni dei secoli XV-XVII annotate e postillate. A questa domanda non potevo rispondere se non esaminando tutte le edizioni delle opere dei vari autori citati dall' erbol'izzatore; e pazientemente mi accinsi a questo lavoro, prendendo nota dei nomi citati nell' indice dell' erbario e riscontrandone tutte le opere registrate nei cataloghi dell' Angelica.

Da questo esame ne trassi che nessuna delle edizioni del Manardo, Ruellio e Odone oggi nell'Angelica, avevano appartenuto all' erborizzatore: non cosi però per alcune edizioni delle opere di Plinio, Fuchs, Teofrasto, Mattioli, Dall'Horto che rinvenni postillate ed annotate ampliamente dalla stessa mano che scrisse l'indice dell' erbario e che numerò le carte e le piante di esso. Ma quella che mi forni la notizia precisa, matematica, del postillatore di quei volumi e nello stesso tempo dell'autore dell' erbario fu un'edizione del Dioscoride del Mattioli (Venezia, 1573) che a pag. 803 reca questa nota: DrIoPTERI, RICAVATO QUI Dal Naturale, CREjO NEL 1583 o 84 DA ME GHIRARdo CIBO, PATRONE DI QUESTO LIBRO.

Una volta messo su questa via, e in possesso di un nome che per il solo esame paleografico comparativo tra il volume postillato e l'erbario mi si rivelava per quello dell'autore di esso, rivolsi le ricerche su questo Gerardo Cibo: e i risultati di esse formano l'oggetto di questa memoria.

Giambattista Cibo - poi Innocenzo VIII - ebbe in giovinezza due figliuoli: Francesco, morto in Roma l' anno 1519 ( $\left.^{1}\right)$ da cui Lorenzo che fu il primo marchese di Massa,

(1) Per la storia della famiglia Cibo, ed i loro possessi in Roma, ricordo alcuni atti notarili riferentisi al palazzo in Borgo - Andreas Gentili et Helias Lomellinus executores testamentarii q. nobilis viri Leonardi Cibo et nobilis domine Franqueta uxor dicti d. Leonardi.... 
e Teodorina che sposò nel 1477 Gherardo Usodimare $\left({ }^{1}\right)$. I figli nati da questo matrimonio furono aggregati alla

vendiderunt nobili viri Joanni de Ancedinia.... medietatem quorundam domorum seu palatii in Burgo S. Petri, iunctam alia medietate hac eadem die vendita dicto Joanni per D. Lodisium Cibo pro pretio duorum millium et quingentorum scutorum de carlenis decem pro ducato monetae antiquae. - Marcus de Mellinis fideiussit pro evictione dictarum domuncularum ut supra venditarum. - Venditio pro I.odisio Cibo facta a D. Augustino Spinola procuratorem dicti Lodisii. Atti Andrea de Carosiis, Notarius, die Vl aprilis 1510. Arch. Capitolino. Script. Arch. R. C. f. 120 et seg. - I confini del palazzo in Borgo erano: ab uno latere domus nobilis Camillae uxoris generosi viri d. Bartholomaei de Ruere, ab alio domus nobilium Vincentii et fratrum de Saxis, ab alio est via publica, ante est via quae dicitur Carreria sancta. - Franciscus Cibo Januensis, magnificus vir, locavit partem cuiusdem domus suae sitae in Burgo S. Petri videlicet ab uno est via Sixtina ab alio latere via Alexandrina discreto viro Bartholomaeo Bachio florentino pro annua pensione viginti duol'um ducatorum de carlenis monetae veteris 1508, die 13 junii. Nicolaus de Bibiena, not. Vol. I, B, fol. 224 Script. Arch. R. C. Archivio Capitolino.

Il Burcardo nel suo Diario oltre Maurizio Cibo, fratello di papa Innocenzo VIlI, ricorda tra i beneficiati Nicoló Cibo, arcivescovo di Cosenza, Lorenzo Cibo cardinale Beneventano, Guglielmo Cibo, cameriere secreto, Matteo suddiacono apostolico, Cristoforo e Pantaleo suoi figliuoli, cugini e consorti del pontefice, oltre molti della casa Usodimare e specialmente di quella di Gherardo marito di Teodorina Cibo. Dei Cibo, beneficati da Leone X, ricordo Andrea, notaro e familiare del papa (19 marzo 1513), Federico, scolare genovese (5 luglio), Geronima, sorella d'Innocenzo, che ebbe la nomina di badessa a vita nel monastelo di S. Sebastiano in Genova (3 settembre).

(1) Di mano di Gherardo Cibo esistono queste note nel manoscritto Vaticano Ottoboniano 3135, c. 233: Anno 1492, 17 julii. - Rev.mo D.no Facius de Sanctoris Viterbiensis emit a R. Camera Apostolica fortilitium nuncupatum la Rocchetta pro pretio duorum millium ducatorum. - Anno 1495 die 17 octobris, praef. D. Facius cessit fortilitium supradictum d.ne Theodorinae Cibo pro ipsa acceptante D. Gherardo Ususmaris dictae d.ne Theodorinae absentis marito et tanquam procurator, pro pretio ducatorum duorum millium, est fol. 26 , et vendidit cum decreto seu breve Alex. VI Pont. quia d. dnus Facius non poterat licite eam vendere, cum de eo litis penderet inter ipsum et quasdam mulieres, sicut in breve supradicto, sub die XX februari 1495, quod penes non est. - Anno 1528 die 3 septembris. D.na Theodorina Cybo vendidit fortilitium Ill.mae Catherinae Cybo Camerini ducissae pro pretio ducatorum duorum millium et trecentorum. 
famiglia Cibo ed ottennero da Innocenzo VIII il titolo di Conti del S. Palazzo Lateranense, la facoltà di legittimare bastardi, dare tutori ai pupilli e creare dottori. Aranino, uno di essi, nato l'anno 1484, custode della rocca di Camerino, molto si distinse nella difesa di questa fortezza in favore di Caterina Cibo, sua cugina, vedova del duca Giovanni Maria Varano, assalita improvvisamente da Mattia Varano che pretendeva alle nozze con Giulia sua unica figlia; sposò Bianca Vigeri della Rovere, nipote di Marco Vigeri vescovo di Sinigaglia. Mori in Sarzana nel 1568, di anni 84. Il suo cadavere trasportato a Massa per cura del figlio Scipione, fu tumulato nella chiesa di S. Francesco $\left({ }^{1}\right)$. Dal matrimonio con la Bianca Vigeri nacquero Gherardo, Marzia, Maddalena, Scipione e Maria $\left({ }^{2}\right)$.

(1) 1568 ottobre 7. Hebbi littere de Scipione mio fratello per le quali mi avvisava mio patre, s. Aranino Cibo essere morto in Sarzana li 16 settembre 1568 essendo entrato già nelli anni 84. Et in quel di medemo fu portato a Massa dove sta sepolto (Gherardo Cibo, Diario, ad a.).

La lapide posta adesso fuori della chiesa medesima, nel muro che riguarda l'orto dietro alla cappella dei depositi dei principi Cibo, è cosi concepita :

ARANINUS CYBO INNOCENTII OCTAVI EX
LINEA FOEMINEA NEPOS INVICTISSIMAE
INTEGRITATIS HEROS CUIUS CERTUM
SPECIMEN DEDIT CUM MATTHEUM VARANUM
CAMERINI ARCIS A DUCISSA CATTHERINA
IPSIUS CONSOBRINA ET AB ILLIUS FILIA CUIUS
AMBIBAT CONUBIUM EJUS FIDEI CONCREDITAE
DOMINIUM MOLIENTEM ARMATORUM MANU
AB EARUM ET ARCIS INIURIA COHIBITUM PROPULIT
HUMANIS REBUS CEDENS GENUAM SE
CONTULIT ET INDE SARZANAM UBI
OCTUAGESIMU QUINTUM ANNUM
AGENS OCCUBUIT M.D.LXIX CUIUS OSSA
SCIPIO EIUSDEM FILIUS IN ECCLESIAM
HANC TRANSFERENDA CURAVIT.

VIANi, Memorie della famiglia Cibo, pag. 79.

(2)

GIAMBATTISTA CIBO (poi Innooenzo VIII)

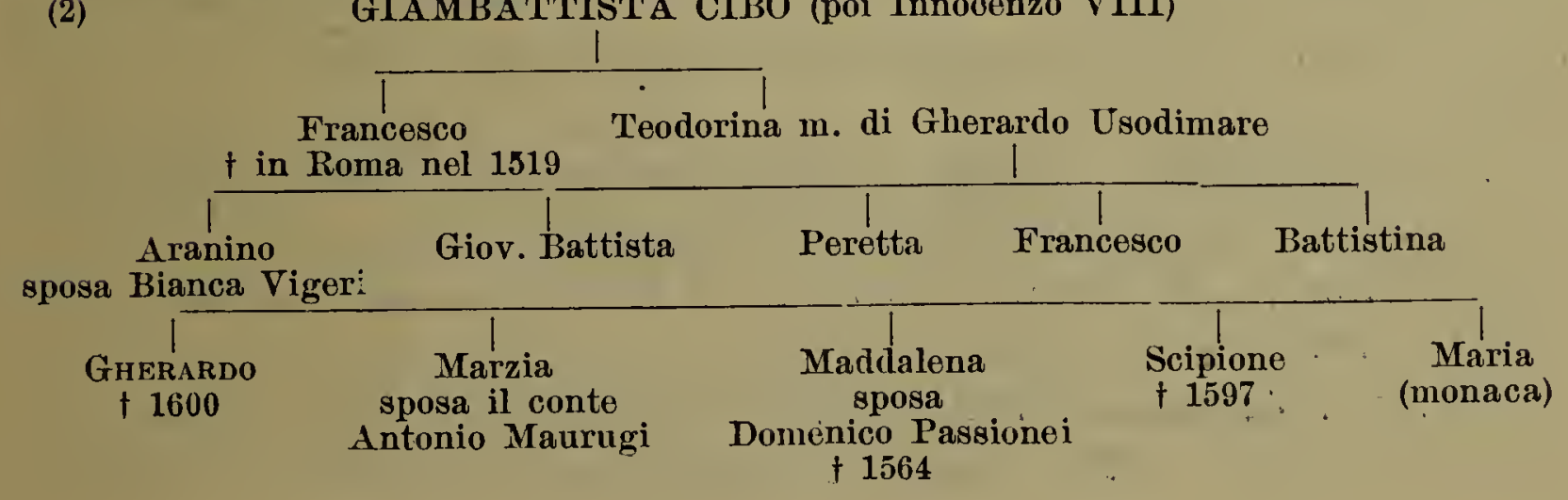


Scipione, nato in Genova nel 1531, dopo avere molto viaggiato (') si stabili, dopo l'anno 1574, in Siena e vi mori nel 1597: e di lui, tra le carte di Gherardo conservate nel manoscritto Vaticano Ottoboniano 3135, conconservasi l'iscrizione che gli fu apposta da Alberico Cibo, principe di Massa $\left({ }^{2}\right)$ : Marzia sposò il conte Maurugi ¿̈i Tolentino $\left({ }^{3}\right)$; Maddalena $\left({ }^{4}\right)$, Domenico Passionei che fu Gonfaloniere di Urbino; Maria, che sopravvisse a tutti e dovè morire vecchissima, fini i suoi giorni nel monastero di S. Agata in Rocca Contrada.

La nascita di Gherardo può fissarsi con certezza avvenuta in Genova l'anno 151\%: erra il Galletti $\left({ }^{5}\right)$ che lo fa nascere l'anno 1520 e morire di sessanta anni nel 1610 , come sbaglia pure il Tasti $\left(^{6}\right)$ che lo fa morire nel 1602 dopo una vita di settanta anni trascorsa in Rocca Con-

(1) Dalle sue lettere al fratello (iherardo risulta che era a Lione nel 1559, a Nimes nel 1561, a Vienna nel 1565, a Massa nel 1568 e 1572, a Siena dopo il 1574. Cit. Lettere nella Biblioteca Comunale di Siena, D. VII, 1.

$\left.{ }^{2}\right) \quad$ SCIPIONI CYBO ARANINI EQUITIS CLARISSIMI FILIO

INNOCENTII OCTAVI PONT. MAX. PRONEPOTI

:QU1 OLIM GENUA ANTIQUISSIMA PATRIA

SENAS SI CONTULIT CIVIBUS IPSIS PROPTER

VITAE INTEGRITATEN CARUS, NON UNO

VIRTUTIS EXENPLO EDITO, LXVI AETATIS ANNO

DECESSIT

M.D.LXXXXVII

ALBERICUS CYBO S. R. I. MASSAE ET CARRARIAE PRINCEPS

GENTILI SUO

posulT.

(3) Di mano di Gherardo Cibo nel mss. Vatic. Ottob. 3135: «Anno 1530 die 7 xbris D. Araninus Gybo constituit dotem duorum millium et sexcentorum ducatorum D. Martiae eius filiae et pro illis duobus millis ducatis, Ill.ma Catherina Cybo Camerini ducissa d. D.ne Martie donavit fortilitium nuncupatum la Rócca di mezzo o di Pier Polo, emptum a D. Theodorina Cybo. Copiato qui de ( 1 di giugno $1589 \gg$.

(4) 1570 febbraio 1, morse a Fossombrone mia sorella Maddalena vidua de M. Domenico Passionei. (CiBo, Diario ad a.).

$\left.{ }^{5}\right)$ Memorie per servire alla vita del card. Domenico Passionei. Roma, 1762, pag. 81 e seg.

${ }^{6}{ }^{6}$ LaELIl TASTll, de situ et origine Rocchae Contratae, libri quatuor, anno 1636, c. 281. (Mss. Biblioteca Romana Sarti, 44. D. 82). 
trada. Più esatto è il Brunamonti $\left({ }^{1}\right)$ che ne fissa la morte al 1600 "l'anno secolare» e asserendo che nel 1540 Gherardo si stabili definitivamente in Rocca Contrada, dà esattamente per la nascita l'anno 1512. Al Galletti, che ebbe in mano le carte del mss. Vaticano Ottoboniano 3135, non sarebbe stato difficile fissare questa data solo che avesse fatto maggiore attenzione e uso piu intelligente dell' elogio funebre letto dal Gilii $\left({ }^{2}\right)$ in morte del Cibo.

Della sua giovinezza ben poco sappiamo: e l'unica fonte è il detto elogio: "Traduxit hic puerilem aetatem et adolescentiam Romae» dice il Gilii «ut optimis moribus et disciplinis in quibus non purum profecit, imbutus, ad dignitates ecclesiasticas, sed magis ad felicitatem consequendam suorum maiorun more aditum sibi patefaceret » e non è improbabile che venisse in Roma seguendo la duchessa di Camerino Caterina Cibo-Varano che vi giunse nel novembre 1526 andando ad abitare in Borgo al palazzo dei Cibo, e tornò a Camerino il 30 gennaio dell' anno seguente $\left({ }^{8}\right)$; fu presente al sacco di Roma, «Verum in ea incidit calamitosa tempora in quibus barbarorum impios furor Romanis opibus inhians, Carolo Borbonio duce, in Urbem summa saevitia irrumpens (proh dolor) illam cepit, diripuit, vastavit, deformavit... quae immanissima spectacula adeo Gherardi et bonorum omnium animos perturbaverunt, perterueruntque, ut felices merito haberentur, qui illinc aufugere, et alio sese reci-

(1) Brunamonti F., Dimostrazione istorica del nobile si antico che moderno stato di Rocea Contrada. In Nuova Rivista Misena, anno IX, fasc. 1, pag. 92 « Cibo, Gherardo di quella sovrana famiglia pronipote di Innocenzo VIII, del 1540, in etá di anni 28 venne in Rocca Contrada, si fece nostro cittadino, piglió pubblici uffici, visse anni 60, e mori nell' etá di 88 l'anno appunto secolare 1600. V'è stata la lapide in S. Franceseo ".

(2) Marci LitiJ Rocchensis philosophi Oratio habita Rocchae Contratae in funere per Illustris Gherardi Cybo Patritii Genuensis prid. non. febrarii MDG. - Questo elogio era tra i manoscritti del card. Passionei di cui fece l'inventario il Galletti: oggi è nel mss. Vaticano Ottob. 3135, c. 215-222.

(3) Fediciangeli, Notizie e documenti sulla vita dí Caterina CiboVarano, Camerino, 1891, pag. 52; Groli D., Certsimento di Roma sollo Clemente VII, Roma, 1894, pag. 9. 
pere possent $»$ e rifuggendo dalle calamità che portò seco il sacco stesso, si trasferi a Camerino presso il duca Giovanni Maria Varano, suo stretto parente «Gherardus igitur Roma profectus ad Johannem Mariam Varanum. cum quo arcta sibi affinitas et necessitudo intercedebat, se se contulit a quo humanissime quidem, ut decuit, in illa calamitate et vastitate exceptus fuit »; dopo passò con Francesco Maria Della Rovere « in omnibus, quae tunc in Gallia Cisalpina bellis gestis, veluti comes et assecla perpetuus secutus est, cui in cunctis periculis, proeliisque praesto fuit, egregiam navavit operam et non minus nobilitate, quam fortitudine gratissimus et acceptissimus omni tempore fuit » che abbandonò poco dopo, cioè nel 1529 per rimanere qualche tempo in Bologna ove frequentò quell' Università e le lezioni di botanica di Luca Ghini. Poi « cum Carolus $V$ imperator adversus quosdam Germaniae regulos, non tam imperii quam catholicae religionis rebelles bellum pararet, in eaque expeditione Paulus huius nominis III pont. max. Alexandrum Farnesium cardinalem nepotem suum magnis copiis mitteret, et Gherardus quoque tunc non postremi ordinis eques, multisque, non minus nobilitate, quam bellicae artis peritia notissimus, nullo stipendio, aut mercede conductus, sed solo tuendae religionis desiderio ductus profectus est, ubi quam strenue, quam constanter, quam fortiter se gesserit, nec tempus, nec locus me ad alia properantem explicare sinunt; sed eo bello feliciter confecto, cum Gherardus vanis mundi et temporum fluctibus agitatus sibi quietem comparare, et quod sibi reliquum erat, totum Christo dicere plane constituisset, ratus inter hasce mundi sordes divinos divinae felicitatis thesauros reperiri neutiquam posse, Marco Vigerio episcopo Senogalliense ejus avunculo hortante, in has nostras oras coeli salubritate et amcenitate haud injucundas, veluti in tutum tranquillitatis portum confugit ».

Mentre il Cibo era in Germania, strane vicende si maturavano per i parenti di lui. Paolo III a Pier Luigi Farnese, suo figliuolo, disegnava già dare stato potente, cominciando con Camerino, antica signoria dei Varano, e perchè appunto allora Francesco Maria della Rovere duca 
d'Urbino gli tolse la preda agognata congiungendo in matrimonio suo figlio Guidobaldo II con Giulia unica figlia di Giammaria Varano e di Caterina Cibo che n'era legittima erede, dichiarò quel ducato decaduto alla Santa Sede. Perciò pubblicati i monitorii sotto pena di ribellione e di perdita del feudo, e mossa accusa contro lo stesso Francesco di avere tentato di sommuovere di nuovo Perugia, non si tosto Rodolfo Baglioni che se n' era impadronito (1 ottobre 1534) fu costretto uscire dalla città, spedi Giambattista Savelli coll'esercito pontificio ad assediare Camerino. Questa minaccia d'armi poteva essere favilla a grande incendio, massime per la pretensione del re di Francia sopra Urbino. Laonde nell' interesse della quiete d'Italia, instarono vivamente i Veneziani si procedesse per la via ordinaria di giustizia civile, e mentre ammonivano il Della Rovere a giustificarsi del fatto imputatogli, esortavano il papa ad « aprire gli occhi e vedere in quali tumulti si trovi Germania, Inghilterra e Francia ». Rispose il papa non potere fare altrimenti da quel che si usa in cose di stato, nè fu senza lunga fatica e molti negoziati da parte dell'Oratore Veneto e dell'Imperiale che s'indusse ad una tregua fino alla venuta di Carlo $\mathrm{V}$ in Italia. Appena celebrato il matrimonio di Ottavio Farnese con Margherita d'Austria, la guerra turchesca diede al papa nuovamente il destro di prendere Camerino. S'interposero anche questa volta i Veneziani in favore di Guidobaldo II che ne era in possesso, ma inutilmente: e questi abbandonato a sè stesso cedette Camerino e ne fu investito Ottavio Farnese. A questa spogliazione avvenuta a danno dei suoi parenti, non poteva rimanere indifferente Gherardo che era al seguito del cardinale Farnese, nepote del pontefice che tanto danneggiava i della Rovere e i Cibo: per modo che tornato in Italia verso la metà dell' anno 1540 , riprese la vita privata, andando. a soggiornare per qualche tempo presso lo zio Marco Vigeri, e ascoltando i savi consigli del vescovo Senigagliese, scelse Rocca Contrada per sua definitiva dimora.

Il Cibo si ritirò a Rocca Contrada l'anno 1540, nell'età sua di anni 28 , e vi dimorò per tutta la vita, assentandosi solo per visitare $i$ parenti.che dimoravano tutti nel- 
l'Umbria o nelle Marche. Una volta ritornò in Roma, l'anno 1553, come rilevasi da una sua nota: " Il di ultimo di novembre mi partii dalla Rocca Contrada assieme al conte Antonio Maurutio mio cognato, per Tolentino, il 16 dicembre 1553 partii per Roma, et il 20 detto gionsi a Roma, dove ritrovai Mons. Rev.mo de Sinigaglia Marco Vigerio da Savona, mio zio, che alloggiava nel palazzo dell'Ill.mo Cardinal Savello a Monte Savello ».

Con l'anno 1553 comincia un suo diario che pervenuto a noi mutilo, è ora posseduto, insieme a molte memorie di lui e di altri illustri arceviesi, dal cav. Anselmo Anselmi che cortesemente lo mise a mia disposizione, di che vivamente lo ringrazio. Questo diario unitamente a molte notizie personali, ne contiene varie ed importanti sulle escursioni che il Cibo faceva vicino e anche lungi da Rocca Contrada per raccogliere piante: ed altre sui lavori e sulle occupazioni predilette sue, che, dopo la botanica, furono la pittura ed il disegno, «tempus perhonestissime contrivit in pingendis enim montibus, vallibus, arboribus, floribus, coeterisque his similibus totus erat ad animi voluptatem, ac virorum praestantiorum quorumdam imitationem, Fabii Maximi nimirum, Hadriani, et M. Aurelii Antonini imperatorum quibus huiusmodi exercitium laudi quoque fuit: tanta autem venustate atque elegantia dilutos colores ex erbis, fruticibus, floribus seminibusque suo marte inventos penna et pennicillo disponebat ut aequalem sibi nullum umquam invenerit neque ipsos Flandrenses hac in arte orbe toto celebres: quae vero ludens effigiare solebat a principibus viris avide admodum expetebantur » $\left.{ }^{1}\right)$.

Di lui il predetto cav. Anselmi, che lo registra tra i pittori Arceviesi del secolo XVI, $\left({ }^{2}\right)$ conserva moltissimi disegni: e nel Diario sopra citato, lo stesso Cibo ricorda ai 27 settembre 1579 «Venne qui alla Roccha Contrata il cavaliere M. Gironimo Ardoino... et mi chiese in prestito il mio libro grande de' paesi a perina, che glie lo pre-

(1) Tasti L., op. cit., pag. 280.

(2) Anselm A., A proposito dei monumenti nazionali della provincia di Ancona. Foligno, Tomassini, 1888, pag. 49. 
stai, con levarli prima molte carte spezzate de' disegni che gli erano dentro » mentre sotto la data dei 22 ottobre 1558 aveva notato: «Da S. Lorenzo in Campo sono ritornato alla Roccha dove ho ritrovato la pietra da macinare colori con il macinello, ogni una de porfido et belle, mandateme da Roma dal nostro Monsignore elletto de Sinigaglia Urbano Vigerio » e sotto li 2 ottobre 1572 "giunsi a Cagli dove alloggiai con i miei nepoti Passionei: la mattina mi inviai per Cassiana dove trovai S. Andrea che mi mostrò il suo libro depinto de herbe quale mi haveria mostrato per prima alla Serra S. Giovanni dove lui era potestà del 1554 ».

Io ebbi la fortuna di rintracciare un' edizione del Dioscoride del Mattioli e un volume di tavole zoologiche dipinte dal Cibo, il primo per il cardinale d'Urbino, l'altro per Francesco Maria della Rovere. Entrambi conservansi nella Biblioteca Universitaria di Roma. Le tavole di zoologia sono legate in un grosso volume $(0.472 \times 0.362)$ legato in pelle rossa con fregi e riquadrature in oro: nelle riquadrature, rappresentazioni mitologiche: ai quattro angoli, busti elmati e laureati: nel centro un'aquila. Taglio dorato. Nel verso della legatura leggesi la nota: Fu fatto al tempo di Francesco Maria primo duca d'Urbino.

Nella prima carta lo stemma dei Della Rovere. Nell' edizione del Dioscoride di Venezia 1568, leggesi nel verso della legatura: "Questo Dioscoride fu colorito e miniato dal Signor Gherardo Cybo, il quale vi fece assai diversi lavori di penna particolarmente nel secondo libro che sono in molta stima. E detto Signore lo donò al Ser.mo Signor Duca di Urbino, Francesco Maria 2..$^{\circ}$. Questa dichiarazione, come pure quella ricordata nel volume di zoologia, sono errate: e lo prova il Diario dello stesso Cibo ove, ai 14 settembre 1573 scrive: " andai alla Badia di S. Lorenzo in Campo a riverire il Signor Cardinale d' Urbino.... et ho ricevuto molte cortesie... et gli piacque per bontà sua accettare da me l'offerta che li feci di volerli colorire un Dioscoride del Mattlioli, tutta volta che mi sarà mandato ». Trattavasi dunque del cardinale e non del duca d'Urbino, e di Francesco Maria secondo e non primo. Il volume del Mattioli gli fu mandato l'ultimo di febbraio del 1574: 
« hebbi il Dioscoride dell'Ill.mo Sig. cardinale di Urbino - segue il diario - con una lettera di Sua Signoria Ill.ma et una di messer Viviano, acciò l'abbia da colorire ». Il 15 ottobre Flaminio Cattabene nomo del cardinale gl' inviò una scatola con 25 ducati d'oro « per comprarne colori » che il Cibo respinse, e un anno dopo, ai 15 ottobre 1575 scrive: «andai a S. Lorenzo in Campo per fare riverenza al signor cardinale di Urbino havendo portato meco tutte le carte del suo libro che ho colorite per mostrarle ». Nel settembre 1578 il cardinale d'Urbino mori, e il volume rimasto nella Biblioteca dei Della Rovere, pervenne poi con le altre opere dell'Urbinate all'Alessandrina di Roma. Di questo volume e della bellezza delle sue pitture, tratta il Bacci nella lettera al Cibo (cf. Doc. III). Di altri disegni di lui fa ricordo una lettera diretta a Francesco Landriani (Doc. IV). Su questi disegni e forse per mantenere la freschezza dei colori, usava il Cibo spalmare una composizione, che credo a base di cera vergine.

In Rocca Contrada diede opera alla fondazione del Monte di Pietà $\left({ }^{1}\right)$ ed il ricordo delle sue elemosine e delle opere carilatevoli era vivissimo ancora parecchi anni dopo la sua morte $\left({ }^{2}\right)$.

Il giorno 8 dicembre 1599 per gli atti del Marchetti,

(1) (Roccha Contrata habet).... et duos, ut vocant, Montes pictatis: unum ubi pignora capiuntur ad egenos sublevandos.... alterum Charitatis, frumenti scilicet sexcentarum salmarum (mensurae genus apud nostros usitatissimum), cuius pars ante festum Nativitatis D. N. J. C., et altera mense maio pauperibus mutuatur. Huic Monti, quam Charitativum appellant, ex propriis facultatibus initium dederunt Gherardus Cybo januensis, Innocentii Papae VIII pronepos, et Camillus Tarucus civis noster, viri in pauperes egenosque ambo liberalitate promptissimi et beneficentissimi, anno christiano 1590 , post illam saevissimam famem, quae apud ltalos pleben invadens impia nece prostravit: triticum namque octoginta aureis pro rubro quod erat paulo amplius sexcentum pondo, emptum a pluribus, a Fabrianensibus praesertim. TAsti L., op. cit., pag. 90.

(2) Pauperes antem tanta semper liberalitate solvit, sublevavitque, ut ipse parcissime vivens, quicquid sibi supererat, ex evangelico praescripto pauperibus crogaret. GiLn, op. cit. Iucundissimam itaque vitam ab omni solicitudine vacuam maximam cum animi tranquillitate producebat, semper liberalis et munificus in omnes. TASTI, op. cit., pag. 280 . 
notaio in Rocca Contrada, dettava il suo ultimo testamento - del quale dò qualche brano perchè mi è stato utilissimo nel ricostruire la vita di lui $\left({ }^{1}\right)$ - e il 30 gennaio 1600 si spegneva placidamente. Nella chiesa di S. Francesco, Marco Gilii, uno degli esecutori testamentarii, ne tesseva l'elogio, che chiudeva dicendo come egli « laetu vultu, laetorique animo (mortem) expectavit: cognoscebat et-

(1) «Al nome di Dio, amen. L'Ill.mo Sig. Gherardo Cibo figliuolo legitimo e naturale del $q$. Ill.mo domino Aranino Cibo, genovese, et habitatore da molti et molti anni in qua della terra di Rocea Contrada, sano per gratia dell'onnipotente Iddio de mente, de senso, vedere et audito, benchè sia infermo, et sia in letto, et iń età senile, costituito etc.... et prima cassa et annulla detto testatore i testamenti fatti da esso Gherardo sotto rogito del q. messer Annibale Pacino, messer Giovanni Beraldo Massaro, et me notaro, per gli anni addietro et de qualsivoglia altro notaro, ecc. Et vole et manda detto testatore che il suo colpo sia seppellito nella chiesa di S. Francesco della Rocca Contrada, nella sua sepoltura, ove esso testatore asserisce essere stata scpelita l'Ill.ma q. Sig.ra Bianca Vigerio Gybo sua madre. » Lascia cinque fiorini a parecchie chiese dentro e fuori Rocca Contrada e all'ospedale, e obbliga i suoi eredi, il giorno dopo la sua morte a spendere «scudi cinque in cose commestibili e dar da mangiare a' poveri per una volta sola »; lascia una vigna, casa e due letti a Maddalena figlia di Mattia da Fossato, moglie di Rocco di Giovanni Battista Garofano della Rocca Contrada, sua serva; all'altra serva, Camilla, scudi venticinque. Lascia ai figli del conte Cristoforo Mauruzi, suoi nepoti e pronipoti della bo: me: dell' Ill.ma Sig.ra Martia Cibo, sorella carnale di esso testatore, e consorte del Sig. Antonio Mauruzi da Tolentino, scudi cento.... Lascia al conte Baldo figlio legittimo e naturale di detta Martia sorella, alli tigliuoli del detto conte Gristoforo Mauruzi per l'altra, la casa fraterna posta in Roma nella piazza di S. Pietro (*), » quasi a rimpetto della fontana et aguglia, quale casa è incorporata con le case dell'Achille Cybo, con un poco di spatio de piedi dieci dalla parte del giardino, in evento che li detti conti vogliano fabbricare et acomodare detta casa.... che vendendosi od affittandosi detta casa si divida il denaro alla metá.... » Lascia alli Sig. Paolo, Innocenzo, Guido e Benedetto, nepoti di esso testatore e figliuoli legittimi e naturali dell' Ill.ma Sig. Maddalena Cybo sorella carnale di esso testatore e moglie del Sig. Domenico Passionei et alli figliuoli della bo: me: del Sig. Silvio pronipote di esso testatore e nipoti di essi Sig. Passionei, la casa che compró il Sig. Scipione in Siena appresso suoi lati e contini, il giardino in Roma con la loggetta, stalla e fienile che riescono nella strada ove

(*) Di questa casa tratta nella lettera al fratello Scipione degli 11 griugno 1581. Biblioteca Comunale di Siena. Cod. D. VII, 9. 
enim, quod, ut philosophus ille dixit: vir sapiens e vita non fugere sed exire debet $\left({ }^{1}\right) \gg$.

La roba del Cibo, comprese le sue carte, andò a finire nel monastero delle Benedettine di S. Agata, ove era monaca la sorella Maria Maddalena: soppresso il monastero sotto il regno italico e non più ripristinato, l'archivio di esso andò disperso, e le carte del Cibo seguirono la triste sorte toccata alle robe del monastero stesso che allora lautamente possedeva in rendita ed in oggetti $d$ 'arte $\left({ }^{2}\right)$.

è il palazzo dell' Ill.mo Carrl. Cesi (") e va dritto nella casa fraterna con il cortiletto congiunto con detto giardino: con il patto che volendo essi Maurugi fabbricare, siano li Passionei tenuti a dare dieci piedi di detto cortiletto ad essi Maurugi... Lascia alla venerabile illustre suor Maria Maddalena, sorella carnale di esso testatore, monaca nel monastero di S. Agata della Rocca Contrada ed al monastero settecento scudi, due pezzi di vigna, mille scudi da esigersi sopra una possessione nel territorio di Jesi, con l'obbligo di dire ogni anno un officio divino per l'anima del testatore e suoi congiunti. - Il testamento fu rogato nella casa degli eredi di Paride Lennciai sita nella contrada Camaldule (oggi Camalduzzi) letto e volgarizzato in presenza del testatore l'anno 1599, mercolerli 8 (licembre, testimoni « Alessandro Cristiano, Giovanni Battista Pictorino, Porzio Francesco Maria Fassono de Urbino phisico dictae terrae, Flaminio Mannelli, Marco Gilii filosofo, Claudio de Marchettis notaro. » (Mss. Vaticano-Ottoboniano, 3135, c. 208-212).

(1) Tandem ad metam sui longissimi aevi placidissime pervenit anno duodenonagesimo, Christi vero domini secundo supra millesimum et sexcentesimum (sic) 3 kal februarii et ad Divi Francisci aedem sepulchrali tumulo est consignatus: ibidemque viri summi laudes Marco Lilio perorante, ab Rocchensibus subiectam memoriam tulit:

D. O. M.

IACET HIC D. GHERARDUS CYBO

JANUENSIS NOBILITATE

INSIGNIS CLARIOR VIRTUTE

VIXI'T ANNOS 88

OBITT DIE 30 JANUARI 1600.

(2) In ultimo, elogio opes, quas non negligendas habebat, perfruendas reliquit sacris virginibus Coenobii Divae Agathae, institutum Divi Benedicti profitentibus, inter quas erat Donna Maria Magdalena Cibo ipsius germana, quae Deo castitaten et vitam ipsa quoque voverat, omnibusque praelucebat ad virtutem. TAstin, op. cit. pag. 281. - Tandem cum vir hic nobilissimus sibi iam prope nonagena-

${ }^{*}$ ) Questa casa era sulla Piazza di S. Pietro, lal lato di porta Cavalleggieri, perchè il palazzo del card. Cesi, passato poi ai Barborini, era dio ro la ehicsetta di s. Michelo e Magno presso detta porta. 
I libri appartenuti al Cibo, e con essi certamente anche l'erbario, come pervennero nelle mani di Angelo Rocca? Nulla di positivo su di ciò: ma non è strano il supporre che questi li avesse o direttamente dallo stesso Cibo o dalla sorella monaca che ne ereditò le carte. Proprio in quegli anni il Rocca, che era di Rocca Contrada, e dovette conoscere il Cibo ed essere con lui in relazione, stava formando la sua biblioteca, e come tutti i bibliofili, dovette andare in cerca di libri ed adoperare tutti i mezzi per possederne. Il Cibo, già vecchio allora, poco o nulla poteva più usare dei suoi, e forse, come dissi, li donò al Rocca: se pervennero in mano della sorella Maria, monaca, questa non dovette troppo farsi pregare per farne un presente al vescovo di Tagaste e al sacrista pontificio. Comunque sia, il Rocca li ebbe, e l'incorporó alla sua biblioteca ove tutt'ora trovansi. Le carte invece subirono triste e peggiore sorte, andarono disperse; molte ne rinvenne e riuni con aifetto di cittadino e compatriotta il cav. Anselmi di Arcevia, altre ne rintracció precedentemente il canonico Salvioni e le inviava al cardinale Domenico Passionei: e sono quelle che oggi trovansi nel manoscritto Vaticano-Ottoboniano, $3135\left(^{1}\right)$.

rio mortem imminere praesentiret ut faciliorem, latioremque ad coelum viam sibi dirigeret, aperiretque in maiorem suae satis amplae hacreditatis partem, nostrum divae Agathae coenobium vocavit, ut totum suae vitae cursum, qui, ut non ignoratis, et a me nunc audistis, in omni aetate, tempore et occasione praeclarissimus fuit, tam honesta pia ac liberali actione concluderet, Gibir, op. cit. - Che le carte del Cibo finissero al monastero, lo prova questa nota che è nel mss. Vat. Ott. 3135, c. 214. «Zia di questo (Cibo) fu la signora D. Zenobia principessa Doria Genovese, come apparisce in una lettera che scrive il Sig. Scipione Cibo al Sig. Gherardo suo fratello, che si conserva in monastero. Peretta, zia di Gherardo, moglie in primo letto del marchese di Finale, si uni poi in seconde nozze con Andrea Doria, donde la parentela dei Cibo con i Doria. Il Cibo non aveva casa propria, anzi dal diario risulta che cambiava spesso di abitazione. In due lettere al fratello Scipione ricorda questo suo stato: in una raccomandandogli un affare " del patrone del suo allogiamento », in un'altra dolendosi di non potere ricevere degnamente il Signor Capponi «perchè S. S. sa che io sto in casa d'altri ».

(1) Il canonico Salvioni scriveva dalla Rocca Contrada al cardinale Passionei in data 4 novembre 1719: « mando la copia del diploma 
A confermare la supposizione che i libri del Cibo passarono direttamente ad Angelo Rocca, sta il fatto che, morto il 6 novembre 1564 il cognato di lui, Domenico Passionei $\left({ }^{1}\right)$, i libri da questo posseduti e molti altri che erano della famiglia Passionei e ne portavano il timbro, furono ereditati dal Cibo e con quelli che costui già possedeva si rinvengono adesso nella Biblioteca Angelica. Da una rapida ricerca fatta nei libri di questa biblioteca, ho ricavato che nove persone della famiglia Passionei, anteriori al Cibo e a Domenico sopra citato, possedevano libri e ne avevano segnato il loro nome sul frontespizio $\left({ }^{2}\right)$ : alcuni recano i nomi di Passionei e Cibo, riuniti. È pertanto ben curioso il fatto che i libri della famiglia. Passionei sieno entrati sullo scorcio del XVI secolo in quella stessa biblioteca Angelica, dove un secolo e mezzo appresso, doveva entrare, per acquisto fattone dagli Agostiniani, la biblioteca celebre e preziosa di un altro membro della stessa famiglia, il cardinale Domenico Passionei, l'infaticabile ricercatore di libri, l' appassionato bibliofilo, a cui le memorie dell' epoca, ricordandone la biblioteca, non risparmiarono e lodi e censure $\left({ }^{3}\right)$.

E anche da notare che in due volumi appartenuti al

conceduto da Federico imperatore alla Casa Cybo, con un foglio scritto dove si esamina la istoria dell'holost che essendo stata ritrovata fra le memorie del sig. Gherardo Cybo, mi persuado possi essere a V.S. Ill.ma grato ». Il foglio scritto è una lettera di A. Bacci al Cybo da Roma «nonis maias 1588 ». - «Gerardo Cybo ex Illustri familia Innocentii VIII, Epistola LXXV primae Centuriae Andreae Bacci Med. ac Phil. ». - Mss. Vat.-Ottob. 3135, c. 206.

(1) De lunedi li 6 novembre 1564 morse Messer Domenico Passionei mio cugnato (CiBo, Diario, ad a.).

(2) Essi sono: Giovanni Francesco Passionei (vol. 1); Benedetto (vol. 56); Domenico (vol. 13); Innocenzo (vol. 8); Orazio (vol. 10): Guido (vol. 4); Passioneo (vol. 1); Mal'co (vol. 1); Paolo (vol. 1). In complesso novantacinque opere. Ad avvalorare questa supposizione sta anche il fatto che molti dei libri di botanica che nell'indice dell' Angelica citato nella nota a pag. 4, sono cosi descritti: «Herbae, arbores vel plantae, ac fructices iconibus representatae, nec non miniatere, ac descriptae multisque tomis comprehensae cum earundem proprietatibus et virtutibus » sono appunto dipinti dalla mano del Cibo.

(3) In un manoscritto delle Satire di Persio, acquistato recentemente dalla Biblioteca Angelica e che porta ora il num. 2666, in uno dei 
Cibo, e di cui parlerò in seguito, trovasi di suo pugno questa nota: «Dato in notola de gennaro 1597. »; orà è azzardata la supposizione che egli compilasse un elenco dei suoi volumi per farlo esaminare dal Rocca affinchè in esso scegliesse quelli che più desiderava di avere, oppure - e ciò è anche più semplice - accompagnasse con detto elenco i libri che donava al Rocca per la nascente sua biblioteca?

Delle opere possedute da Gherardo Cibo, da lui postillate e oggi conservate nella Biblioteca Angelica, segnalo qui soltanto quelle di cui feci uso per i confronti paleografici con l'erbario distinguendole progressivamente con una lettera dell' alfabeto che servirà per i richiami e le note all' indice dell' erbario.

A. Mattioli A. Il Dioscoride. - In Vinegia, appresso Vincenzo Valgnisi, M.D.XLVIII. - Annotato e postillato dal Cibo, come ne fa sicura fede, oltre il carattere la nota a pagina 745: "Di queste pagate is ne ho avuto forsi 4 o 5 anni sono mandatemi da! fattore di $M$. Domenico Passionei mio cugnato, che fu ritrovata in quel di Cagli» ed il Passionei, come vedemmo, aveva in moglie Maddalena Cibo sorella carnale di Gherardo. Le note e postille sono in inchiostro nero e rossastro, di varie epoche. Il frontespizio e molte piante sono dipinte (cf. pag. 5, 14, $20,22,45,302,303,338,340,343,366,369,370,383$, $384,437,449,457,460,463,466,473,475,478,479,483$, $484,488,489,490,506,507,510,512,518,519,521,525$, $538,545,553,566,571,588,589,597,604,607,610,620$, $643,644,645)$ : alcune note dànno notizia che il Cibo era anche raccoglitore di pietre (cf. pag. 742) «Pietra asia » una polvere sottile che si puo chiamare fiore, trovai io in una grotta sopra de' quei sassi a Agnano, loco del Sig. marchese di Massa, presso a Pisa tre o quattro miglia, che era sutilissima, e del colore non era nè bianca nè

risguardi leggesi: « Nos hunc nostrum codicem comparavimus Callii e Comite Beroaldo indocto homine, possussor autem bibliothecae quam collegerat Abbas Guastellensis doctissimus Baldus. Proh dolor ! ex bibliotheca nobis innotuit postquam expilata tam fuerat ab insigno illo circulatore maniobiblico et fure cardinali Passionaco ». 
verde, nè gialla nè bertino, ma era quasi nel mezo de tutti questi colori et ce n'era assai. La grotta è vicina alla strada maestra, a piè del monte, e dentro à una vena d' aqua »; pag. 747 «Pietra Morochto 》 potria essere quella pietra che Pieragostino mi ha donato 3 di sono, che lui la prese nella isola de Malta nella grotta dove si dice che si fermò S. Paolo. Questa è bianca, tenera et segna quasi come il gesso, de X.re 1565. Pieragostino è el fattor che gic serviva $M$. Domenico mio cognato » pag. 750 «Pietra ophite de X.re $1565 》$ Questa ophite penso che possa essere una pietra che io tre di sono trovai sul monte della Justitia della Rocca Contrata, quando dalla cima si cala per una valle sassosa che dirittamente arriva a certe case chiamate le piaggie: la trovai appresso dove si cavano le pietre non so se per fabricar o per far calcina: questa è ponderosa, tra il negro e bertino, con macchiette piccole rosette, verde, biancastrine e bertine, con certi segni minuti che si trovano.... molto piu che la marchesita fa foco col focile et non poco.»

B. Mattioli A. Commentarii secundo aucti in libros sex Pedacii Dioscoridis... - Venetiis, ex officina Erasmiana, V. Valgrisi, 1558. - Sul frontispizio vi è il timbro con la leggenda: «Bibliothecae Passioneae ». Ha poche note e postille del Cibo. Quasi tutte le figure sono dipinte. Furono asportate recentemente parte delle pagine 18 e 512 .

C. Mattioli A. I Discorsi nelli sei libri di Pedacio Dioscoride, ecc. - In Venetia, appresso gli eredi di Vin. cenzo Valgrisi, M.D.LXXIII. - É questa l' edizione del Mattioli che più servì al Cibo. In alto del frontespizio reca: Ghirardo Cibo, nel basso: Benedetto Passionei, e il timbro di questa famiglia con la leggenda. La lettera dedicatoria del Mattioli è datata da Innspruch, 1 aprile 1568: e questa opera, tra quelle del Cibo nell'Angelica, ci fornisce notizia delle relazioni tra l'autore e il Cibo stesso. A pag. 44 parlando del Cneoro il Mattioli dice: «Una pianta disegnata di sua propria mans et con arte sottilissima colorita, mi mandò già da Roma il gentilissimo Signor Gerardo Cibo, la quale tanto in ogni sua parte si rassomi- 
glia al Cneors bianco di Theoprasto che veramente non si puo negare che non sia quella istessa come par la sua figura, la quale è qui, si può far vera coniettura. » $\mathrm{E}$ a pag. 788 ripete: "Il Cneoro di Theoprasto con tutte le note mi fu mandato dal magnifico Signor Gerardo Cibo.»

Nella Tarola molti nomi di piante sono segnati con due punti, e riscontrati coll'indice dell'erbario è risultato che tutti i sègnati cosi si riferiscono a piante contenute in esso. Alla lettera $G$ della stessa tavola, dalla medesima mano che scrisse il Gerardo Cibo del frontespizio, è ripetuto questo nome e ricino ad esso i- numeri 788, 40: e infatti alla pagina 788 , linea 40 , il Mattioli fa menzione del Cneoro inviatogli dal Cibo.

Non è però questa la prima edizione del Dioscoride del Mattioli nella quale si citi l'invio del Cibo, ma bensi quella pure tatta in Venezia dal Talgrisi nel 1568 nella quale le note surriferite trovansi rispettivamente alle pagine 51 e 1328. Questa edizione però non è posseduta dall'Angelica.

Il Mattioli nella tavola ta uso spessissimo dei richiami e vedi a voci e forme simili, e in quei casi manca la citazione della pagina, per modo che il ricercatore è costretto ad una doppia consultazione prima di trorare l'indicazione roluta: il Cibo ad evitare questa noia, vicino a quei $r i$ chiami mise a penna il numero della paginazione a cui si riferivano, e questo, mentre risparmiò a lui, servendosi del volume, tempo e fatica. fu a me prezioso e sicuro riscontro per l'identificazione dell' erbario rispetto alla numerazione delle carte e piante di esso. Le postille sono in inchiostro nero e rossastro: che siano della stessa mano lo dimostrano quelle a pag. 11 1 . 335, 335, ove trovansi riunite, con identica scrittura, e differente colore d'inchiostro. Alle molte note, delle quali alcune riporterò ad illustrazione dell' indice dell' erbario, univa anche l' esame scrupoloso dell'opera: a pag. 228 corregge un difetto del tipografo e scrive sotto le incisioni: "porpore e buccine, buccine queste, difetto dello stampatore; porpore sono queste », e similmente a pag. 524 ne corregge un altro, scrivendo: "il dauco II: questu. pianta fu posta nella seconda specie del precedente contra il volere del. Matiolo ». 
D. Theophrasti, De historia et causis plantarum - Parisiis, E. Gourmonotum, 1529. Bollo della famiglia Passionei. - Le postille del Cibo cominciano a pag. 116 e sono dello stesso inchiostro rossastro usato per annotare l' edizione del Mattioli (C) e correggere e numerare le carte dell' erbario.

E. Fuchsius, Historia plantarum, S. n. n. - Ha le incisioni tutte dipinte, e le postille in carattere grande, simile all' indice dell' erbario. Sono qui con costante continuità segnati i rimedii contro la podagra, malattia di cui soffriva il fratello di Gherardo, Scipione, e fors' anche lui stesso.

A questi volumi che serviranno a fare dei riscontri con l' erbario, se ne possono aggiungere alcuni altri a titolo di curiosità e pure appartenenti al Cibo.

Dall' Orto Garzia. Due libri dell' historia dei Semplici, aromati et altre cose che vengono portate dall'Indie Orientaii pertinenti all'uso della medicina. - In Venetia, 1582. -- Sulla 1." guardia reca questa nota: «1586, Ghirardo Cibo. Questo libro con un altro simile che vog(lieva) mio fratello lo ricevuti di giovedi li 5 de giugno 1586 mandat(imi) dal S. Cavalier Gironimo Ardoino, portatomi da Paolo gia hebreo: il costo dell'un(o) paoli $5 \frac{1}{2}$ (de l'altro .5 $1 / 2$ ) che tra tutti dua sono paoli ondici ». Nel verso: D.ta in lista ge(nnaro) 1597.

Sannazzaro G. Arcadia. - Vinegia, per Nicola d'Aristotile detto Zoppino, 15:30. Sul frontispizio in alto: Ghirardo Cibo; a metà: Data in notola de gennaro 1597. In basso: Di Benedetto Passionei, e timbro della biblioteca di questa famiglia. Con vignetta dipinta.

Certamente appartennero alla Biblioteca Passionei, e forse al Cibo, l' edizione delle Epistole di Plinio (Bologna 1502) e l'Expositio anatomiae Mundini cum additionibus Carpi (Bononiae, 1508) pure conservate nell'Angelica: in esse rinvengonsi postille anteriori al Cibo, ma alcune mi sembrarono di sua mano.

Il Cibo usava di scrivere in due maniere assolutamente 
differenti tra loro: o in carattere grande, di scrittura chiara e nitida, o in carattere piccolo, rotondo, irregolare. Questa seconda maniera è la più frequente. I confronti paleografici dovevano essere necessariamente duplici, perchè l'indice dell' erbario è in carattere grande; la numerazione delle piante, delle carte dell'erbario e i nomi, sono quasi tutti in carattere piccolo. A questa duplicità di riscontro, servirono mirabilmente i libri postillati ed annotati da lui ove rinvengonsi note copiose nelle due differenti scritture. Come saggio di confronto, con il carattere grande dell'indice si possono tenere a riscontro le opere segnate:

A) numeri della tavola alle pag. 285, 691, 709;

B) carte $a .2$, a.2; $3.5 ; \beta .6$;

C) carte biiij, eij, eiij, fiiij, e i numeri tutti della tavola.

E) pag. 4, 68, 126, 128, 215, 304, 333, 337, 347, 355, $417,419,431$;

con il carattere piccolo dei numeri e dei nomi delle piante nell' erbario le opere segnate:

A) pag. 295, 310, 409, 525, 709, 742, 745, 747, 750;

B) aiiij, $\gamma 1$, * 4; pag. 195, 432, 435, 436, 438, 454, 480, 490;

C) pag. $129,150,325,455,545,567,569,573,600$, $619,663,686,691,790,791,803$;

D) pag. 253 ;

E) pag. $45,46$.

Naturalmente queste citazioni riguardano luoghi ampiamente postillati e tali, per la loro complessività, da offrire facile ed ampio esame tra i due caratteri: chè sarebbe stato inutile ed ozioso citare qui tutte le pagine postillate dal Cibo. Chi poi volesse abbreviare tale comparazione può limitarsi a confrontare:

D) pag. 253 cauda vulpis, con le identiche parole nell'erbario vol. I, c. 247 ;

C) pag, 521 «Smirnio di Candia» con le identiche parole nell' erbario, vol. III, pianta 644 ;

C) tavola c. liiij il nome di Gherardo Cibo, con la parola: absinthium dell' erbario vol. I, c. 3 ;

B) le pärole Anthera (c. a. 2); pes lecticae clinopodium (c. $\beta, 5)$; verbascum (c. $\beta, 6)$, con le identiche parole nell' indice dell' erbario. 
L' erbario messo assieme dal Cibo consta realmente di quattro volumi: un altro volume, che nulla ha che vedere coi primi, e che sta ora a rappresentare un quinto volume, non è, secondo me, che il primo tentativo di un erbario. Questo volume isolato, senza numerazione di carte $(\mathrm{mm}$. $260 \times 195)$ contiene 515 piante numerate progressivamente più una in fine non numerata. Le piante sono in cattivo stato di conservazione: parecchie di esse totalmente perdute, altre ridotte a ben poco. A renderle cosi contribui la primitiva legatura del volume: l'erborizzatore non calcolò l' aumento di spessore che le piante avrebbero portato mano mano che l'inseriva, il dorso si ripiegò all' indentro forzando il volume ad uno stato anormale e molte di esse, in quella costrizione violenta, si spezzarono e frantumarono. Era un erbario mal riuscito: ed il Cibo dovè abbandonare l'idea di cavarne qualche utile perchè non risulta ne compilasse alcun indice, e le stesse carte non numerò, contentandosi di numerare solamente le piante. Questo tentativo di erbario che a mio parere risale a qualche anno innanzi a quello contenuto in quattro volumi, rese avveduto l'erborizzatore che ripetè il lavoro in modo tale da ottenere risultati molto più concreti e pratici. I fogli del secondo erbario, la loro numerazione e l'assegnazione delle piante tutto fu preparato innanzi che il Cibo vi inserisse di fatto le piante che ideò di mettere per ordine alfabetico: sui fogli scrisse il nome della pianta che vi avrebbe poi agglutinata, molti però rimasero in bianco e le piante che oggi sembrerebbero a prima vista mancanti non furono mai collocate. Fece poi legare i quinterni a volume: ma già l'ordine che si era proposto eraglisi alterato per l'inserzione di piante di cui non aveva preveduto o prevenuto l' acquisto, e la numerazione delle carte fu rinnovata.

Vol. I. (mm. $311+216)$. I quinterni sono segnati progressivamente dall' $A$ alla $Z$ : le carte numerate da 1 a 230 , le piante da 1 a 316. Mancano, ed evidentemente non vi. furono mai collocate, quelle ai numeri:

46. Allium ursinum

137. Argemone.

52. Alsine species

141. Armoracia 
61. Alipon

65. Amarantus luteus

68. Ammonum

91-92. Angelica inodora

93. Anysum

110. Aphaca vera

111. Apios vera
171. Balausticum

217. Cairella

230. Cannabis

236-238. Carduus

241. Carum rerum

247. Cauda vulpis

316. Colocynthis

113. ....? ? ...

I nomi delle piante sono scritti ora in carattere grande ora piccolissimo: i numeri in inchiostro nero o rossastro.

Vol. II. $(\mathrm{mm} .311 \times 216)$. I quinterni sono segnati progressivamente dall' $A A$ alla $Y Y$ : le carte vanno dal numero 1 al 230, le piante dal 310 al 637. Dalla carta 7 la numerazione passa alla 12, ma l'ordine numerico delle piante non è interrotto: una lacuna verificasi nella numerazione dal 541 al 547: la pianta 408 è duplicata. Mancano le piante ai numeri:

328. Corylus

337. Crocadylium

373. Cyprinus rotondus

380. Damasonium

381. Daucus (?)

382. Daucus vulgaris

Daucus creticus

403. Dorycnium

415. Elephoboscum
402. Faba sativa

467. Filix foem.

476. (Foeniculum syl. Hippomerathron)

490. Gentiana maior

542. Harundo

582. Hieracium parvum

584. Hippophestum

Vol. III. (mm. $311 \times 216)$. I quinterni sono segnati da aaa a zzz: con la carta $2: 32$ comincia la segnatura Aaaa e va sino al $B b b b$. La numerazione delle carte va da 1 a 25̌1: quella delle piante dal 638 al 1038. Mancano le piante ai numeri:

684-685. Lotus urbanas

205. Peliurus

917. Paparer album

$1010 . \ldots ? \ldots$

1011. ...? ? . .

1013. ...? ? ...

Vol. IV. $(\mathrm{mm} .311 \times 216)$. I quinterni sono segnati Cccc $D d d d d$. La numerazione delle carte va da 1 a 227: quella delle piante dal 1038 al 1347. Sono duplicate le piante 1061, 1074, 1183, 1243, 1271: mancano quelle segnate: 
1089. Satyrium

1136. Seseli creticum

1160. Sison
1213. Symphitum petreum

1229. Thapsia vera

1336. Vitis alba.

Non tutte le mancanze dell'erbario, ossia le carte bianche, possono dirsi mancanze di piante: perchè molte di esse, e sempre per quell'ordine che il Cibo sul principio si prefisse ma dovette poi abbandonare, se mancano ove dovevano originariamente collocarsi, trovansi altrove. E infatti l'indice spesso reca l'indicazione di due carte, l'una, priva della pianta, l'altra, con la medesima pianta agglutinata. Eccone i numeri:

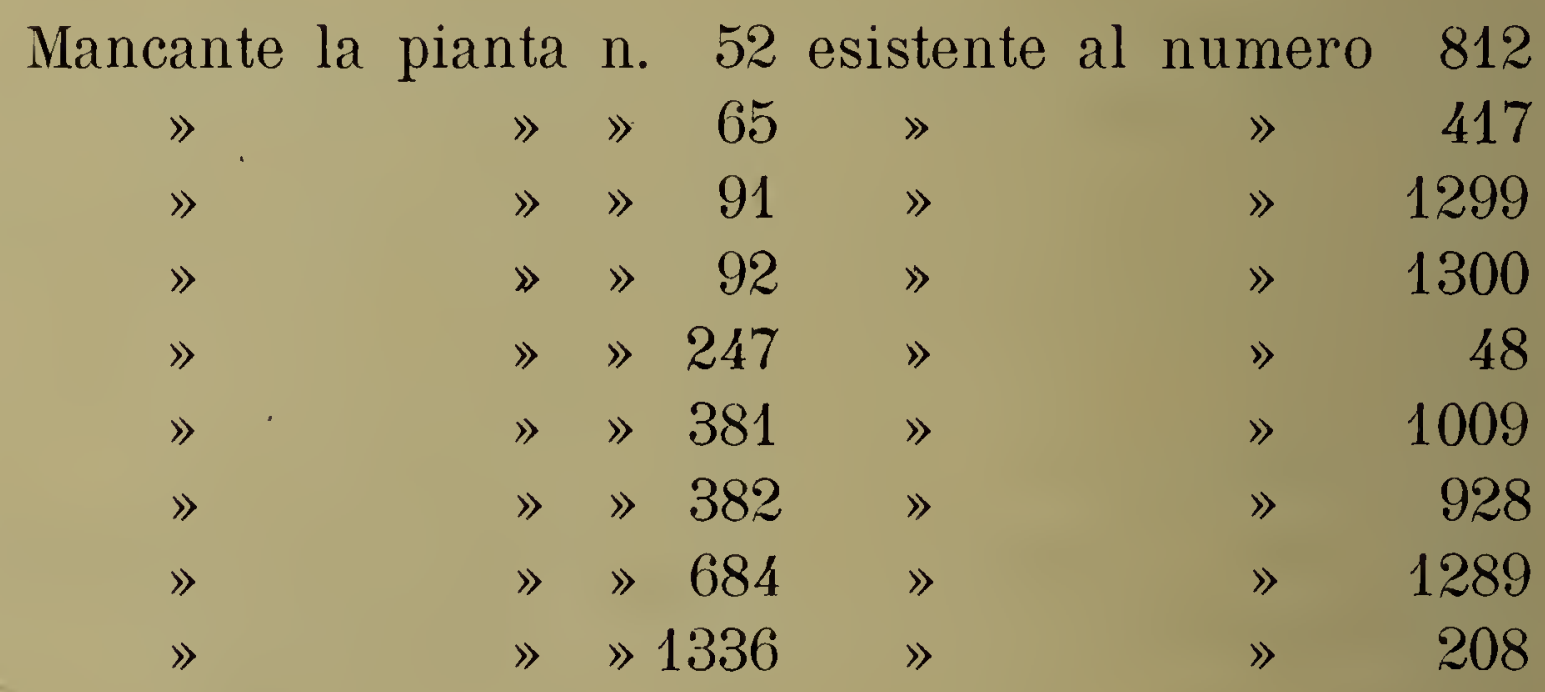

Per modo che le piante elencate e non messe, si riducono da cinquantuno al numero di quarantadue, cosa davvero ben esigua di fronte alla vastità dell' erbario.

Ogni pianta essiccata fu agglutinata o incollata sopra un foglio. Ogni esemplare è numerato e spesso trovasi indicato col nome latino adottato dal Cibo, corrispondente a quello adoperato dagli autori più in -voga in quei tempi e citato poi nell'indice dell' erbario. Parecchi i nomi volgari. Sopra ogni foglio è agglutinata una pianta sola; ma non raramente anche due o tre specie: ed allora ciascuna è indicata coi numeri corrispondenti. La completa ignoranza di questo erbario, che per tre secoli rimase inoperoso e obliato, giovò moltissimo al suo mantenimento ed alla sua integrità. Le piante classiche che in altri erbarii troppo ammirati e studiati scomparvero, qui furono conservate, ed oggi ogni sottrazione sarà resa difficile dall'accertamento numerico delle piante da me fatto e riportato più sopra. 
Per rapporto al numero delle specie, quelle rappresentate nell'erbario del Cibo, supera quanto di tali collezioni del secolo XVI ci è pervenuto. Risulta infatti da dati certi che l' erbario di Andrea Cesalpino contiene 768 specie, quello Estense 182, l'erbario del Girault 313, quello grandissimo del Bauhin circa 4000 (di cui solo una metà giunse a noi), quello del Retzemberg 746, e del Rauwolf circa 400: mentre questo del Cibo ne contiene 1347 che unite alle 515 del volume senza indice, fanno complessivamente il bel numero di 1862 specie. É pertanto, dopo l' erbario dell'Aldrovandi, molto più limitato nella varietà essendo esso composto in massima parte della flora bolognese, il più vasto che a noi sia pervenuto e che rimonti ad epoca tanto remota.

Quando Gerardo Cibo mise assieme il suo erbario? Quantunque non sia facile a prima vista dare una risposta matematicamente precisa a tale domanda, perchè del Cibo nessuno trattò, e per esso mi trovai a brancolare nel più fitto buio, pure spero di giungere a conclusioni abbastanza serie e precise.

Gerardo Cibo era in Roma l'anno 1527 e doveva esservi venuto in una delle tante gite fattevi da Caterina CiboVarano negli anni dal 1524 al 1527: dopo il sacco se ne fuggi presso il padre a Camerino e vi rimase fino alla morte di Giovanni Battista Varano avvenuta nell' agosto dell'anno stesso. Passò poi con Francesco Maria della Rovere, parente di sua madre, e con lui milito in Lombardia e rimase fino all' anno 1529, allorchè per la coronazione di Carlo V, il Della Rovere prefetto di Roma, si recò a Bologna a prestare omaggio al papa ed all' imperatore, e nei capitoli della pace generale, gli fu confermata l' investitura del suo stato.

Era allora legato di Bologna il cardinale Innocenzo Cibo, zio di Gherardo, e dimorava pure in Bologna, ove rimase fino all' anno 1532, l'altro zio Lorenzo Cibo marchese di Massa. Con uno dei due, e molto probabilmente con Lorenzo, di cui più tardi lo ritroveremo nuovamente ospite, rimase Gherardo: ed è in questo periodo - malgrado non 
possa per questo recare prove innegabili - che egli studiò nell' Università Bolognese, nella quale sino dall' anno 1528 insegnava l'illustre botanico Luca Ghini. Che egli venisse in Bologna nell' anno 1529 è provato: ed è del pari provato - come si vedrà - che nell'anno 1532, allorchè abbandonò questa città, egli avesse cognizioni botaniche tali da permettergli osservazioni e note che un profano in materia non avrebbe potuto fare.

Nell'agosto del 1532 Carlo V scriveva da Ratisbona alla vedova di Giovanni Maria Varano, Caterina Cibo, come gli stesse a cuore si combinasse il matrimonio tra Giulia figlia di Caterina ed erede del ducato di Camerino, con un figlio di Carlo di Lannoy principe di Sulmona. La Cibo non si mostrò favorevole al desiderio dell' imperatore e gli rispose evasivamente: incaricando di portare la risposta Aranino Cibo padre di Gherardo, che condusse seco il figlio. Passati per Trento si spinsero fino ad Ingolstad e poi a Ratisbona, dove allora dimorava l'imperatore per la dieta apertasi il 17 aprile dello stesso anno.

Di questo viaggio si hanno due ricordi nei libri postillati dal Cibo: in $C$ pag. 129 alla Betula annota: "che $i$ contadini di Trento lo chiamano bedollo » a pag. 663 alla pimpinella maggiore: "Io l'ho veduta in Germania per la campagna o di Ratisbona overo di Iglestad ». È dunque abbastanza chiaro che il Cibo partendo nel 1532 per il viaggio di Germania doveva avere studiato botanica: e che studiasse giovanissimo è anche provato da una lettera di Andrea Bacci - riportata in appendice - che ricorda come egli ne fosse cultore ab usque primis annis: e nel periodo corso dal 1529 al 1532 , il Cibo aveva appunto dai diecisette ai venti anni. Che egli studiasse con Luca Ghini, sarebbe abbastanza provato dal fatto che dimorando in Bologna niun altro poteva avere per maestro: pure nelle sue note ei ne lasciò una prova, diremo cosí, morale.

Nell'indice dell'erbario, vicino ai nomi delle piante, il Cibo riporta spesso la citazione di autori di botanica; ma queste citazioni sono composte del solo cognome, abbreviato nel modo più strettamente possibile (cf. Pl. (Plinio); 
Mat. (Mattioli); Fuc. (Fuchsius); Man. (Manardo); solo il nome del Ghini che è riportato un' unica volta alla $L y$ chnis agria è scritto così: Dni Lucae Ghini; quasi a significare la reverenza dello scolare per il suo grande maestro $\left({ }^{1}\right)$.

E poi evidente, se si confronta l' erbario del Cibo con quello del Cesalpino, pure scolare del Ghini, la grande uniformità tra loro nella classificazione e denominazione delle piante, tanto che i due erbarii, se ignoti ne fossero gli autori, dovrebbero necessariamente giudicarsi come provenienti da una sola scuola: ed è la stessa classificazione del Cibo che, in più vasta scala dell' erbario, adoperò poi il Cesalpino nella sua opera De plantis.

Nel 1534 Gherardo Cibo, trovavasi in Agnano, presso Pisa, ospite di Lorenzo Cibo marchese di Massa, che vi si era ritirato appena morto Clemente VII $\left({ }^{2}\right)$, e li faceva collezione di pietre «pietra asia, polvere sottile che si puo chiamare fiore, ritrovai in una grotta sopra de quei sassi a Agnano, loco del S. marchese di Massa presso a Pisa tre o quattro miglia »: poi più nulla potei sapere di lui fino all'anno 1539 in cui va con il cardinale Alessandro Farnese a Carlo V. Il Farnese aveva studiato in Bologna nell'epoca nella quale vi dimorò pure il Cibo, e nel 1531 era scolare di Ugo Boncompagni: nulla di più semplice che accogliesse nel suo seguito nullo stipendio aut mercede conductus - come dice il Gilii - uno che

(1) Il nome dei Cibo si trova spesso nelle rubricelie degli studenti universitari di quell' epoca. Di un cugino di Gherardo si fa menzione in quella dell' Università di Pisa, ove ai 25 ottobre $15 / 8$ notasi: «Dnus Octavius D. Laurentii Ciibo scholaris artista solvit pro sua matricola soldi $12 »$. Era costui il figlio del marchese di Massa presso il quale Gherardo aveva dimorato in Bologna e in Agnano. - Andrea Cibo lesse molti anni medicina nello studio di Pelugia: mori il 17 maggio 1576 di anni 83. Cf. CRIsporti, Perugia Augusta, Iib. 3, pag. 335; RenazzI, Archialiri pontificii, I, 330.

$\left(^{2}\right)$ Questi beni furono dati da Lorenzo De' Medici a Franceschetto Cibo per dote di Maddalena sua moglie. « El magnifico Lorenzo....... la maritò (Maddalena) al signor Francesco Cibo et li diè in pegnio per la dote lo Spedaletto, le mulina di Ripafratta e beni nel contado di Pisa, luogho detto Agniano come si vede a libri della decima ». - Garte Strozziane, filza 22, c. 74-80. 
doveva considerare come antico compagno di studi e colto amico.

Alessandro Farnese nepote di Paolo III fu inviato a Carlo $\mathrm{V}$ in occasione della cerimonia per la morte dell'imperatrice avvenuta il 1 maggio 1539. Trovavasi allora l'imperatore nel convento dei Gerolamitani della Sysla a due miglia circa da Toledo, ed ivi il cardinale ai 18 giugno gli fece le condoglianze del pontefice, e due giorni dopo, la relazione dei pubblici negozi commessagli pure dal papa. Mentre il Farnese s'intratteneva a Toledo scoppiò l' insurrezione di Gand: e Francesco I fece significare all'imperatore che se voleva traversare la Francia onde abbreviare il cammino per recarsi nelle Fiandre, n'avrebbe avuto il maggiore piacere del mondo. Dopo non brevi trat. tative ed esitazioni, Carlo V il 10 novembre parti da Madrid, il 28 era a Baiona, il 12 dicembre a Loches ove si incontrò col re di Francia, e il 1 gennaio 1540 entrava in Parigi. Avevano seguito l'imperatore onde sollecitare la concordia tra i due potenti monarchi, Marcello Cervini già fatto vescovo di Nicastro e cardinale di S. Croce, e il cardinale Farnese. Partito il 7 gennaio da Parigi, Carlo V entrò nei Paesi Bassi a Valenciennes il 21, a Gand il 14 febbraio ove rimase sino al 30 aprile, promulgazione della sentenza di alto tradimento. Il legato Farnese - e con lui il Cibo - rimase a Gand fino al 9 di maggio: il 17 era a Saint-Germain, il 27 giugno in Roma da dove scriveva al cardinale Cervini.

Nella prima metà del giugno 1540 il Cibo abbandonava il Farnese che tornavasene in Roma e si ritirava in Rocca Contrada. Seccato dal genere di vita nomade che aveva menato dalla sua infanzia; disgustato; lui fervente cattolico e nobilissîmo d'animo, delle lotte religiose di quei tempi e degli intrighi della politica di cui la sua famiglia e i suoi congiunti con l'elevazione di Paolo III al pontificato avevano inteso sì gravi angustie, amante dello studio e della sua libertà, nessun luogo poteva più attrarlo che il paese ove erasi portata a vivere sua madre. Aveva costei, nel 1528, ceduta la Rocchetta a Caterina Cibo (cf. pag. 6); il marito era lontano quasi sempre da lei e pur lontane due sue figliuole: il primo occupato nelle 
condotte militari, le seconde accasate a Fossombrone ed a Genova; non le rimaneva che tornare presso i suoi, i Vigeri, allora stabiliti in Rocca Contrada e presso l'unica delle figliuole rimasta nubile e monaca nel convento di S. Agata. E Gerardo che doveva di essa essere amantissimo, tanto da disporre nel suo testamento d'esserle sepolto vicino, si riuni cosi alla madre ed alla sorella, e mai più si mosse da Rocca Contrada.

La parte del diario del Cibo, pervénuta fino a noi, comincia con l'anno 1553: manca tutto quanto poteva dare notizia dei suoi studi e sopratutto di quando avesse cominciato a raccogliere ed agglutinare piante. Da esso tolgo alcune poche notizie riguardanti la raccolta del Cibo:

1557, maggio 22 .... andai insieme con Gaspare Marchetti et con un altro da Sigillo.... in cima al monte.... dove trovammo gran copia di peonia, massime dalla banda che scopre il mare: trovammo similmente gran copia di narcissi. Et in certe balze de' sassi all'andare in su trovammo grandissima copia di gigli azzurri e fioriti havendo l'odore degli altri irios. Ma nelle radice non si sentiva alcun odore. Et portammo una soma de ribos trovati in valle ombrose che si trovano nell'ascendere il monte.

1566, settembre 30. De domenica..... si montò sopra Monte Nero per la strada della valle di gorgo nella quale a un miglio dentro messer Alfonso Ceccarelli $\left({ }^{1}\right)$ da Bevagna e medico di Gualdo vidi il titimollo mirsinite.... lo quale per essere di colore simile alla ruta anzi più chiaro si vedeva benissimo. E nasceva poco lungi dalla strada, et ne pigliammo una pianta.

1568, aprile 28. Andammo al Monte detto Sassoferrato... dove trovai il narcisso che già fioriva, et similmente trovai quella sorte di martago che fa il fiore di colore tra il verde, giallo e rosso, e tutto piticchiato de' punti paonazzi scuri, che a questo fine andai di questa stagione a

(1) È costui il celebre falsario di documenti e carte antiche, decapitato in Roma nel giugno 1583. 
questo monte sapendo che in tal tempo è solito fiorire. Trovai il croco martis ma sfiorito. Et de tutte queste ho prese e portate. Sopra il detto monte ho ritrovato la stellaria che non credevo ci nascesse.

1569, ... ... montammo al monte Regedano.... e al monte contiguo molto più alto.... dove sono herbe più notabili, et ove si ritrova assai herba luccia dal fior turchino.

1570, giugno 22.... mi partii dalla Rocca per l'Alvacino... . a mezza montagna trovai il cardo bianco e bello che anchor non haveva fiorito, alto forsi due cubiti, con el gambo grosso un deto et vacuo, che io credo sia la spina bianca.

1573, giugno .... poi andammo alla Badia Santa Croce..... nel monte di detta badia ci trovai copia di elleboro bianco fino appresso alla fonte, dove ne colsi un poco.

1573, luglio 11. Andassimo a Monte Cucco, dove su la cima trovai il nardo montano, molto bello con radice sode e basse, et ne ritrovammo per tutto all' intorno della cima, ma non già in basso. Trovammo ancora assai stellaria.... trovammo l' herba paris.

Nei 18 detto andai alla Trinità..... dove su le sue mura viddi da cinque o sei piante di quella bella pianta che fa fronde et fiori assai simili a quelli del rododafne anchorchè assai minori et fiori spicati. Per i prati all' alto trovai gran copia di filipendula fiorita, copia grande de carlina, eufragia infinita e molte piantè de effodillo e stellaria.

1573, luglio 23.... andai al Castello di Passilupo..... trovai gran copia dell' herba gatta, j. nepeta.

1573, agosto $3 \ldots$ passai per Passilupo e mi fermai a raccogliere la bettonica in quel luocho dove sta tanta copia de enula campana. 
1575, giugno 26. Andai al monte del Sassoferrato.... nel prato più alto ... fu trovata da me della scorzonera, bella con radice grossa et alcune longhe un palmo et più, et grosse alcune come un grosso bastone, piene de succo latteo. Trovai ancora.... una sorta de geranio cioè piè colombino con le radice curte et grossette quasi come mazzole.... i fiori erano piccoli con un certo colore tra l'incarnato e pavonazzo, le radici di fuori negre: cioè di dentro bianche con certi circoli purpurei et di sapore astringente.

1576 , giugno $17 \ldots$ andai sul monte Catria e trovai assai fegatella.

1577, ottobre $1 \ldots$ nella selva delle grotte (del Massaccio) nasce assai bonifatia.

1581, ottobre 4. Andai a S. Gironimo di Passilupo a pigliare dell' angelica in fondo al romitorio.

1582, agosto 14. De venere con Gaspare Marchetti e con Berardo Bianchi andai alla grotta dell'Aracoeli per ritrovar la lunaria odorata o greca che non trovammo...... giunsimo alla cima del monte cogliemmo della sassifragia dalle fronde simili al fenocchio... colsi della luccia maggiore... li alla grotta non potei trovare di quella pianta che vado trovando e credo possa essere la mirris. Ci ritrovai una gran pianta di solatro maggiore... et per quel sito ci sono più piante di herba caprina che mi è stata cara havercela ritrovata.

Se il diario ci fosse pervenuto nella sua interezza, le notizie che riguardano la raccolta del Cibo, forse risalirebbero all'epoca della sua dimora in Bologna (anni 15301532), perchè prestissimo - ed è il Bacci che lo afferma - egli cominciò le peregrinazioni e le gite alpinistiche e botaniche. "Nisi quantum assiduo labore, ac studio conati sunt cui similes aliquas tantae confusionis verruncare et esplanare semitas, quod tu ab usque primis annis ad hunc validam senectam praestitisti, qui hoc solo studio ac amore indagandae naturae plantarum et stir- 
pium non contentus peragrasse diu diversas Italiae terras ac salebrosos Apennini subire et perlustrare montes mira ad luce dexteritate studiisti.... ». Il Cibo dopo il 1540, anno in cui si ritirò in Rocca Contrada, non si mosse che per gite o piccole escursioni e sempre nelle Marche ed Umbria: e quei viaggi da lui compiuti in giovinezza e nelle varie contrade d'Italia ai quali accenna il Bacci debbono certamente riferirsi ad epoca anteriore all' anno 1540 .

Nè pregiudica a questo asserto l'osservare come nell'indice dell' erbario siano citati autori che pubblicarono le loro opere verso la metà del secolo XVI ed anche dopo, e nell'erbario si conservino piante la cui importazione è certamente della seconda metà del secolo stesso: l'indice è di molto posteriore alla formazione dell' erbario: e questo, ideato e cominciato con il sistema delle piante in ordine lessicografico, fu poi ampliato e completato, tanto da sconvolgersi quasi del tutto l'ordine primitivo con le piante che pervenivano al Cibo, o che egli medesimo si procurava nelle sue escursioni. A questo sconvolgimento dell' ordine ideato, rimediò il Cibo compilando l'indice dei quattro volumi di esso: non tenendo affatto conto dell'altro volume che, mal riuscito, rovinato nella sua conservazione non distrusse forse per quell' affetto o feticismo che si ha talvolta per i lavori proprii, anche se cattivi: volume che rappresenta, almeno a mio parere, la prima ed informe concezione di un erbario.

Nella Flora romana $\left(^{1}\right)$ i proff. Pirotta e Chiovenda, trattando di Andrea Laguna, osservano: «Aggiungeremo qui la notizia, che riteniamo nuova, che il Laguna fece un erbario, giacchè a proposito della Pśoralea bituminosa egli scrive: (Laguna, Dioscoride, pag. 342). «Hallase gran copia del Trebol bituminoso inuto a S. Juan de Latran en Roma, de donde yo mismo cogi una planta que aun tengo entre otras conglutinada ». A nessuno sfuggirà l'importanza di questa notizia: già abbiamo veduto che il Laguna asserisce di essere vissuto parecchi anni in Roma anche prima del 1546, laonde può il Laguna competere

(1) Pirotta, Chiovenda, Flora Romana, Roma, 1901-902, fasc. I-II. 
il primato nella formazione degli erbarii, che finora, come tutti sanno era attribuita al Falconer, il quale pare abbia fatto il suo tra il 1540 e il 1547. Però noi a questo punto osserviamo che ambedue furono grandi peregrinatori nell'Europa e ambedue frequentarono in Italia le lezioni di parecchie università, e in modo speciale, nell' università di Bologna, quelle di Luca Ghini, il quale si sa di certo che raccoglieva ed agglutinava piante. Non ci pare quindi improbabile che da quest'ultimo maestro ambedue appren. dessero quella maniera di conservare le piante che venivano raccogliendo nei loro viaggi. Anche Meyer $\left.{ }^{(}{ }^{1}\right)$ viene a queste conclusioni ».

E questa una forte scossa al primato del Falconer, ed un grande vantaggio per coloro che asseriscono essere stato il Ghini il primo ad agglutinare piante. Nè minore importanza ha quanto può congetturarsi sull' epoca nella quale il Cibo cominciò il suo erbario.

Come dimostrai, egli studiò in Bologna, certamente col Ghini, nel periodo corso dal 1530 al 1532, cioè molto tempo prima che il Falconer e il Laguna ne frequentassero le lezioni: il Falconer poi, passava da Ferrara portando il suo erbario tra gli anni 1540 e 1547 : ma se è vero quanto asserisce il Camus $\left({ }^{2}\right)$ che egli in quell' epoca fosse di ritorno dai suoi lunghi viaggi e che cinque anni dopo il 1547 era in Inghilterra citato dal Turner tra i migliori e reputati botanici inglesi, è lecito supporre che passasse da Ferrara più verso iI 1547 che il 1540. Però, e questo nella migliore delle ipotesi per i fautori del primato del Falconer, pure accettando l'altro estremo limite, cioè l'anno 1540, il Falconer passava da Ferrara quando il Cibo era assente dall'Italia già da quasi un anno, e tornandovi, si ritirava a Rocca Contrada senza toccare Bologna e Ferrara. Egli evidentemente non conobbe il Falconer: e non da lui, ma dal Ghini dovette apprendere e mettere in pratica, molto prima del Falconer stesso, l'arte di conservare ed agglutinare le piante: tanto più che nell'erbario si

(1) Geschicht. der Botanik, IV, 271-273.

( $\left.{ }^{2}\right)$ Historique des premiers herbiers, Malpighia, IX, 7, Gènes, 1895, pag. 13. 
riscontrano varietà di piante che egli stesso forse riportò dai suoi viaggi, come ad es., la betula che si conosce essere non una pianta coltivata, ma trovarsi quasi esclusivamente nel Trentino, dove appunto passò il Cibo sullo scorcio dell'anno 1532, notando che colà essa è chiamata dai contadini bedollo. Ne vale l'osservare che nello stesso erbario sianvi piante non rinvenibili in Italia e che dovettero necessariamente essergli inviate: perchè questa osservazione riguarda piante rare o di allora recente scoperta, mentre la betula è una pianta comunissima e tanto di poco valore da potersi senza danno trascurare in un erbario, e, data la difficoltá delle comunicazioni d'allora, non preoccuparsi troppo per possederne un esemplare.

Facendosi poi ad esaminare le marche della carta usata dal Cibo, si trova una nuova conferma alle mie deduzioni. Il volume che attualmente è il quinto dell'erbario, e che io dissi una prima e mal riuscita concezione di esso, reca costantemente la marca del toro, che ricorre in quella data forma, nelle carte di fabbricazione veneta - probabilmente Padova e Treviso - sullo scorcio del secolo XV e principio del secolo XVI (cf. a. 1482, 1503-1504); gli altri quattro volumi hanno le marche dell' àcora sormontata dalla croce che rinviensi in carte veneziane dagli anni 1502 al 1544: dei tre monti con croce e cappello senza fiocchi produzione della medesima epoca: del giglio chiuso in un cerchio che ritrovasi identico in carte degli anni 1539-1552; dell' àncora senza anello e cerchiata, che riscontrasi negli anni 1538-1541, e della testa di moro, forse marca Fabrianese, unica nella serie, pure della prima metà del secolo XVI $\left(^{1}\right)$.

Come appare chiaro, la carta usata dal Cibo è nella massima parte produzione di cartiere venete, ed evidentemente egli ne fece l'acquisto in qualche centro - non asserirò fosse Bologna - alimentato dalla produzione veneta, e questo prima dell'anno 1540, nel quale anno egli si ritirò a Rocca Contrada, perchè se l' erbario l' avesse cominciato dopo tale epoca, la carta dovrebbe recare tutte

(1) Ringrazio vivamente i Sigg. proff. A. Venturi e A. Zonghi per le notizie fornitemi sulle marche delle carte. 
marche Fabrianesi, come il luogo di produzione quasiunico che allora fornisse i mercati delle Marche e dell'Umbria di cui Fano e Perugia ne erano gli emporî. Non pretendo con ciò di sostenere il valore preciso e scientifico della rispondenza tra gli anni di fabbricazione delle carte e l'impiego fattone; ma è indubitato - e lo provano tutte le recenti monografie sulle marche della carta che quasi sempre trovasi una perfetta rispondenza di anni tra la scritturazione di manoscritti o documenti e la produzione della carta adoperata, e se rinviensi alcuna differenza essa non risulta superiore ai tre o quattro anni. Se si riflette poi che essendo molti i bisogni e scarsa la produzione, la cosa non recherà meraviglia.

Non mi sembra quindi più sostenibile l'ipotesi del Camus favorevole al Falconer: comunque sia, l'erbario del botanico inglese è scomparso, e quello dell' Aldrovandi rappresenterebbe nella storia della botanica il più antico ed il più importante documento di questo genere $\left({ }^{1}\right)$, poichè date le ricerche del Camus, del Saint-Leger e del Mattirolo il suo inizio risalirebbe all' anno 1551. Innanzi ad esso viene oggi a porsi questo di Gherardo Cibo: della. sua importanza e valore botanico tratterà, illustrandolo nelle singole specie, il prof. Penzig, che volonterosamente volle assumersi tale incarico; io, povero profano in botanica, tentai solo di ricostruirne la storia e ricercarne l'anonimo autore.

Di Gherardo Cibo, come botanico, niuno fa menzione: nell'accurato lavoro sulla Flora romana $\left({ }^{2}\right)$ che è la più recente e minuziosa opera che sulla storia della botanica e sui botanici siasi fino ad oggi pubblicata, il suo nome mai apparisce. Eppure fu in relazione e riscosse la stima dei migliori botanici suoi contemporanei, quali l'Aldrovandi, il Mattioli, il Bacci: e questo silenzio su di lui è solo spiegabile dal non avere egli prodotto e pubblicato qualcosa che lo tramandasse ai posteri, almeno nel frontespizio di un libro. Forse di lui rimase qualcosa inedita?

(') Mattirolo O., Illustrazione del primo volume dell' erbario dell’Aldrovandi, Genova, 1899.

$\left.i^{2}\right)$ Op. cit. 
Le sue carte e le sue robe andarono disperse: e le mie ricerche, vôlte specialmente alle biblioteche ed archivi dell'Umbria e delle Marche, riuscirono infruttuose: forse - e questa mi pare la migliore delle induzioni - non si occupò di nulla in particolare, astrazione fatta dell'erbario e della riproduzione in pittura delle piante e dei luoghi artistici e pittorici dell' Umbria. E in questo fu perfettamente simile al Falconer, del quale il Turner scrivera: There haue bene in England, and there are norv also certain learned men: whych haue as muche knowledge in herbes, yea, and more then diverses Italianes and Germanes, whyche haue set furth in prynte Herbelles and bokes of simples $\left({ }^{1}\right)$.

L'Aldrovandi ricorda, due volte di seguito, il Cibo nel suo Catalogus virorum qui mea studia adiuvarunt $\left({ }^{2}\right)$ : l'una, chiamandolo «Cardinali de Cybo nepos» e l'altra «Genuensis Gerardus de Cybo nepos cardinalis de Cybo »: e certo dovette essergli grato per l'invio di piante 0 agglutinate o dipinte.

Dei rapporti col Mattioli, che conobbe per mezzo del fratello Scipione, ho lungamente accennato innanzi, e molto più ne potranno dire le lettere che pubblico in appendice (Doc. I-II): essi cominciarono l'anno 1563 con l'invio di molte piante dipinte dal vero, e subito il Mattioli, che forse non fece in tempo a citare e ringraziare il Cibo nelle sue Elistole medicinali edite a Lione nel 1564 ove tratta diffusamente del Cneoro, ne fa speciale e replicata menzione nell' edizione del Dioscoride del 1568. Forse la corrispondenza col Mattioli continuò, ma di essa nulla

(1) Turner W., A new herball, London, 1551.

(2) Mss. Aldrovandi N. 110 nella Biblioteca Universitaria di Bologna. In questo stesso mss. nel « Catalogus virorum qui visitarunt Musaeum nostrum et manu propria subscripserunt in nostris libris Musaei » troviamo notizia di tre personaggi di Rocca Contrada che visitarono il Museo Aldrovandiano e certo lo fecero per sollecitazione del Cibo. Essi furono: « Eques auratus artiumque et medicinae doctor Fulgentius Belo de Rocca Contrata » del (quale il Cibo nel suc) Diario registra le gite botaniche insieme compiute e la morte: « $\mathrm{D}$. Victorius Philippinus Theologiae doctor » e «Petrus Manellus studiosus » forse parente di quell'altro Mannelli che figura come testimone nel testamento del Cibo. 
giunse a mia notizia; solo da una lettera di Gherardo al fratello Scipione, si rileva che, morto ii Mattioli, ne ebbe il ritratto che « ricevette con grande piacere ».

Del carteggio con Andrea Bacci, rimase una lettera: quantunque unica, il suo contenuto è importantissimo, perchè pur tenendo conto della forma eccessivamente laudatoria, dà un'idea chiara di quanto quel botanico, se non illustre certo non ultimo tra i suoi contemporanei, apprezzasse e stimasse il Cibo (Doc. III).

Altri ancora lo aiutarono nel mettere assieme l'erbario: nell'ottobre 1574 gli manda alcune piante il cardinale di Urbino, e nel gennaio dell'anno appresso, in una postilla al Dioscoride del Mattioli, egli ricorda: «I fiori di questo sambuco sono roscetti et di colore assai simile alla pietra corniola: nel territorio del Castelficardo ne nasce, presso certe acque, da dove mi fu portato da Francesco herbolaro da Sorbolongo ».

In appendice pubblico alcuni brani di lettere dello stesso Cibo (Doc. IV-XII), essi servono maggiormente a lumeggiare la figura di questo ignorato botanico, del quale, come risultato delle mie modeste ricerche, sono lieto di avere aggiunto il nome, non inglorioso, alla storia della botanica in Italia.

Roma, 1902. 


\section{DOCUMENTI.}

Doc. I.

Molto magnifico signor mio

Prego V. S. che se bẹn mi accusarà di negligenza, non me vogli però tenere nè per discortese nè per inhumano, se più presto che hora io non rispondo alle sue de' XX di marzo, quali ricevetti il passato agosto, inviatemi dal mio M. Gio. Angelo Crotto: voglio ohe, mercè sua, me creda ció non essere intervenuto se non per legitime cause, dalle quali me son trovato così occupato, che in sei mesi non ho nè scritto lettere nè risposto a veruno. Troppo sarebbe lungo il narrarle ogni cosa, ma persuadendomi che V. S. mi crederà, posporrò per hora questa parte, et solo attenderò, se ben tardi, a risponder alle sue cortesissime et amorevolissime lettere, certificandola che le mi sono state di.non poca consolatione, vedendo fuor d'ogni mio pensiero d'aver ritrovato un amico di tanto valore. Però non poco me ritrovo obligato a M. Gio: Angelo, che di così onorato acquisto è stato cagione. $\mathrm{Ho}$ inteso con piacere infinito dell' opera rara che ha fatta in pittura delle piante el signor Gherardo suo fratello, la quale mi pare fin di qua vedere come in uno specchio di purissimo cristallo. Volesse Idio ch'io la potesse vedere visibilmente, chè più chiaro mi sarebbe che veder qual'altra si voglia cosa che dilettar mi potesse: peró poscia che la lontananza ne veta ch'io non possia vedere il tutto, prego V. S. che almeno voglia far ch'io ne vegga tanto quanto la me scrive, cioè un paro di quelle figure fatte con sì bello artifizio et con tanto. giuditio et diligenza: che facendolo, oltre che io ne le resteró obligato, mi darà causa di mandarle qualche dozzena delle mie figure nuove grandi cavate con non poca fatica dal vivo, le quali sono fino al numero di mille, e tutte saranno nel Dioscoride nuovo che hora si stampa a Venetia. Se io sarò buono a giovare all' opera del suo signor fratello, tenga V. S. per certo che io le saró liberalissimo di quanto sarà in potestà mia, imperò che io non sento refrigerio maggiore che giovare et servire a huomini virtuosi. Però voglio che V. et sua S. facciano di me quel capitale che farebbono loro stesse di se stesse insieme, che sempre me ne ritrovaranno pronto et apparecchiato per servirle. Et qui facendo fine, senza fine me le raccomando.

Di Praga, alli 20 di Dicembre 1563 di V. S.

affettionatiss. serv.

Pietro Andrea Mattioli.

(Indirizzo)

Al molto magnifico signor mio osserv. ${ }^{\text {mo }}$ il Sig. Scipione Cibo a Marsilia.

(Biblioteca Comunale di Siena, Mss. miscell. D. VI, 7 , f. ${ }^{\circ}$ 3). 
Doc. II.

\section{Molto magnifico signor mio}

Ho ricevuto questi giorni passati la lettera amorevolissima di V. S. insieme con le figure delle piante fatte per mano del molto magnifico suo fratello, le quali come cosa rara, et da me molto desiderate, mi sono state tanto chare quanto dir le possa, perchè in vero me par che siano tanto vive et naturali, che più non vi si possa desiderare: et massimamente che vi si discerne una leggiadria che dà loro l' ornamento di quei paesetti ameni, che non si discerme nelle naturali. Onde le dico che se ben da molti ot diversi luoghi mi sono state mandate piante colorite, tanta differenza veggio tra queste et quelle, quanto si vede dal piombo all'argento o per dir meglio all'oro. Me ne furno mandate di Costantinopoli, già furno tre anni, cinquanta'in foglio reale, le quali furno tenute tutte per una cosa bellissima, ma appresso a queste (tanto può il paragone) paiono una cosaccia. Di qui adonque vengo in cognitione che il libro del sig. suo fratello sarà il più bello di quanti se ne trova al mondo, et prego Idio che a lui dia gratia di finirlo et a me tanta vita che possa vederlo. Risponderò a sua signoria più presto' ch'io me sia sleggerito d'alcune occupationi che me tengono come prigione, et intanto V. S. le potrà mandar questa, acció resti sicuro che ho ricevuto il suo cosi bello et honorato dono, per il quale me li tengo non poco obligato. Et qui facendo fine resto pregando Iddio che et a lui et a V. S. dia ogni contento, et le bascio (!) la mano.

Di Praga, alli 24 de' giugno. 1565. di V. S. servidore

Pietro Andrea Mattioli

(Indirizzo) Al molto magnifico signor

mio ossermo il Signor Scipione Cibo

Vienna. In sua assenza al signor

Giovanni Angelo Crotto agente del signor conte di Fiesco.

(Bibl. pred. Mss. miscell. D. VII, 2, c. 15).

Doc. III.

Gerardo Cibo ex Illustri Familia Innocentii VIII Epistola LXXV primae Centuriae Andreae Baccij Medici ac Philosophi.

In qua examinatur Historia Holostii apud Dioscoridem variis opinionibus. 
Cum simplicium historia, ac rei plantariae sylva (ut cum vulgo Medicorum loquar) amplissima sit, et diversarum ab invicem specierum vix explicabilis similitudo, Gerarde Vir Illustris, non mirum, ut cum a doctissimis ac eximiae in hac parte peritiae vivis fuerit adeo excussa, adhuc tamen inacessibiles habeat saltus, et inextricabiles ambages. Nisi quantum assiduo labore, ac studio conati sunt cui similes aliquas tantae confusionis verruncare et explanare semitas, quod tu ab usque primis annis ad hanc validam senectam praestitisti, qui hoc solo studio ac amore indagandae naturae plantarum et stirpium non contentus peragrasse diu diversas Italiae terras ac salebrosos Apennini subire et perlustrare montes mira adhuc dexteritate studuisti perspicuis coloribus ac picturis tua manu naturales ipsas plantas ita effingere ad vivum, ut in tanta hodie luce Matthioli, Clusii, Dodonaei (cum pace dixerim aliorum et Patavinorum doctorum) ex his potissimum yconibus, quos ad me proxime misisti plantarum abstrusae notitiae, et Holostii inter has, praeclarum mihi visus sum habere argumentum de tuo appicto Dioscoride, cuius iam fama celeberrimus es, nihil esse pulchrius, nee praestantius. In quo vero perhumaniter a me requiris quid sentiam de Holostio. Equidem praeter hanc tuam de eo picturam, quam adeo exquisite ad naturam delineasti, eo magis tuum laudo iudicium, quod dum varias de eo invenerim nostrorum hac aetate interpretum positiones, vix tamen ullam mihi videtur referre genuinam speciem. Quippe Dioscorides, quem habemus in hac parte naturae (Galeno teste) antesignanum lib. IIII. cap. X. « ijoscov inquit, quod et cis:\%ov herba est pusilla, humi incum« bens, trium aut quatuor digitorum folia habens, et viticulas, «Coronopo similes, aut gramini, gustu adstringentes, radice alba "praetenui quae in capillamenti usque speciem, longitudine qua« tuor digitorum nascitur in collibus ». Affatim, inquam, his notis tua haec respondet plantula, exiguis foliis, gramini proximis, sapore adstringenti, radicibus capillaribus, ceu Nardi spica protensis et odoratis in collibus ac fabulosis nascens locis. Deficit autem quod haec viticulosa non est, immo foliis gramini proximis exilibus, ac nodulis distincta, habet vero insuper et tenues culmos et spicas in apice, Ristide similes, idest inanes semine. Et praeterea Plinius addit inter praecipuas notas, hane lib. XXVII, cap. X ab Etimologia Holostion dici, quasi sine duritia, ex adverso hoc est per antiphrasym, quia scilicet folium cum duritie habeat, et forte scabrum, haec tenuibus est. In reliquis imitatur Plinius Dioscoridis descriptionem, quanquam ipsum non citat, vel quia non legit iam ab Augusti usque et M. Antonii aetate scriptorem, vel ut facile unus ex alio transcrihit multa, eadem accepisse videtur a praece- 
dentibus ante Dioscoridem scriptoribus. Deficit adhuc magis Matthiolus, qui ut in primis eius editionibus Holostium nec cognovit nec appinxit, ita in uitimis reposuit Plantam valde tuo Holostio adsimilem, eique a Mutinensi quodam Medico transmissam, sed apocripham. Unde eam nullis describit notis, sed quam ex relatu illius appingit foliis graminis, et culmis apice non spicatis sed a medio sursum alatis cum flosculis Odontidi aemulis, ut dentes serratulis, quos Monachinas hodie appellant, vel cum spicis divisae sint in quolibet culmine, Aegilopi proximis, hoc (ni fallor) Aegilopis potius species nequaquam Holostium. Sunt et qui a spica parva plantaginis, caudae muris nomine putant Holostium humilibus et ipsa foliolis, per quam angustis arctibus (?) coliculis, qui in spicam desinant tenuem herbaceam, caudae muris aemulam. Caeterum et haec longe differens ab Holostio est.

Minime vero omnium pro Holostio acceptanda est Clusii et Dodonei positio, quorum novissime legimus de natura stirpium commentaria, qui plantaginis angustifoliae (?) ponunt speciem, foliis minoribus angustioribus, lanugineque albida, incanescentibus, humi procumbentibus, inter quae seni aut quini pediculi attolluntur seorsum versus, flosculis gravidi, iisque spicatim digestis, semine cum maturaverint in minutis valvulis quale plantaginis radice longa, tenui et lignosa in aridis nascitur. Quae notae vix ullae leguntur in Holostio Dioscoridis caret viticulis, et insuper pediculis abundat ac spicatis floribus, quae in Holostio non leguntur. Quare sententiam quasi revocans Dodoneus, haud verum hanc admittit Holostium. Mihi vero ea figurae specie relata, quam pro Holostio appingit. Onosma, haec potius videtur Dioscoridis, quae Anchusae aut Ecchij species est, foliis quatuor digitorum longitudine, et unius latitudine, mollibus, humi stratis, sine caule, sine flore, sine semine, radice oblonga, tenui infirma, rubescente: gignitur in apricis. Quale memini me vidisse copiosum, ac colligi iuxta salebrosas Potentiae fluminis ripas cum proficiscens Matilica Septempedum irem, sine culmine (ut dictum est) nec flore nec semine praeditam. His ergo reiectis opinionibus quam proprie speciem dixerim Holostium, nullam magis quam hanc tuam video congruam. Nec obstat eam carere viticulis, attolli vero pediculis et spicatis aristulis. Nam saepe Dioscorides et alii auctores ex notis omittunt aliquas, et cum Dictamnum similiter descripserit sine flore et semine, ex adverso flosculis, ut perspique niteat purpureis iamdiu in nostris Italiae hortis videre est. Similitudines porro in plantis vite decernendis saepe fallit indicium. Nam qui hodie Herbarii ex officina Plantina prodierunt libri, qui innumeras a similitudine sub eodlem genere plantae multiplicant species, et graminis 
triginta, vel amplius figuras non sine taedio ignarae iuventuti hanc sub Hordei adnectunt genere. Ut etiam Aegilopem, festucam, Zeam sterilem, et Holcus apud Plinium lib. itidem XXVII cap. X. In saxis (inquit) nascitur siccis, aristas habens in cacumine tenues, culmo quale ordeum restibile, unde et Ristidam hanc quidam vocant. Huius ergo minorem speciem et sterili spica videtur tua plantula aemulari, ut etiam radice odora est, et arida, desiccandi vim habet cum adstrictione, Galeno teste. Quamobrem adrupta cum vino eglutinanda ulcera in decoctis probatur, quo nomine quidam, ut Matthiolus, Pilosellam a chyrurgis vocatam putant. Nec subticenda cum Plinio et Dioscoride proprietas, quod Holostium cum carnibus coctum, eas cogit. Atque hanc equidem censuram, Gerarde optime, in tuam reporto gratiam. Vale. Romae Nonis Maias M.D.LXXXVIII.

Tuus ex affectione Andreas Baccius. m. pr.

(Bibl. Vatic. - Cod. Ottob. 3135 c. 224).

Doc. IV.

Ill.mo Signore e P.rone mio Osservand.

Adesso ho ricevuta una sua con la inclusa di messer Gironimo Ard[oino] et cusi per il suo mandato gli mando de quella rosetta che dice messer Gironimo, la quale per essere da dieci anni che fu fatta ha perso di colore: perchè questa sorta de rosette mantiene il suo bel colore quasi per uno anno, et: poi si svanisse: niente di manco per l'effetto che si ha de operare: credo che sarà buonissima, tanto più quanto che la sarà temperata per l'opera con acqua di gomma dove sia un poco de allume de roccho. Messer Gironimo soleva havere una laccha chiara che per questo effetto è buonissima. Se di questa Rosetta ne havessi havuta più, più gle ne haveria mandata, et me despiace non sia de quella bellezza che si converria pur sarà V. S. Ill.ma contenta accettar il mio-buon animo.

Ho fatto cinque pezzi di disegni come in Senigaglia gli promessi, quali gli mando per il presente suo mandato. Me rincresce non saperli far meglio sì per la satisfattione sua come che a me saria anchora de maggiore honore. Sono tre in carta azzurra de chiaro et de scuro: uno in carta bianca et l'altro colorito in carta azzurra. Anchora che siano goffi potranno" fursi far questo effetto, che quando la li vedrà si ricorderà che gli sonno affezionato servitore come fui sempre.

Me saria charo che V. S. Ill.ma si degnasse farmi sapere si li haverà ricevuti, che volendomi far scrivere potrà farla dare a mes- 
ser Domenico Passioneo mio cognato. E con questo humilmente gli baso le mano pregandola si degni comandarme dove La cognoscerà sia bono a fali (!) servitio che l' haverò sempre a grandissimo favoro. La prego anchora si degni basar le mano per mia parte al Sig. Conte Pietro, al quale son servidore de core.

Dalla Roccha contrada

alli 21 de giugno 1562.

Di V. S. Ill.ma

Servitor Gherardo

Cibo.

(Tergo) : Allo Ill.mo Sig. Conte

Gio. Francesco Landriano

Sig. et Prone mio Osserv.mo

in Urbino

(Arch. di Stato, Firenze. Archivio dei Duchi d'Urbino. Class. I, Div. G, Filza 268, carte 87).

Doc. V.

Dalla Roccha Q.ta li 29 de Gennaro 1579.

.... ho visto la memoria che mi date di più quadretti vi feci, et di un paesino, che sta nel libro de herbe, che si ben mi ricordo, sta depinto nella figura dell'Elleboro bianco. La radice che mi havete mandata non è quella del milium solis, che amazza i vermi essendo questa una radice con molti filamenti sottili, che deve andare per il traverso della terra, che penso sia il milium solis maggiore dal fior celeste, et grande come le viole salvatiche, cioè litospermo: quello che voi desiderate cognoscere fa ben le fronde assai simile al maggiore dal fior turchino, ma le fa più negre, più dure, et più aspro: con il fior piccolino, et bianchiccio, i fusti diritti più duri, più grossi: et la radice grossa come il deto minore, quando più et quando meno che va per diritto sotto terra, senza filamenti: negra di fora, et qualche volta rossigna, che tenge di rosso le mani : et di dentro bianchiccia: et dolce assai. Nasce nei terreni sterili, et brecciosi, come in greppi et apresso le giare dei fiumi, dove sia herba, et arburi.

Se in simili lochi porrete cura per adesso ne potrò ritrovare i fusti secchi con delle perlette di sopra, ma per ciarirvi (sic) del vero, bisogna cavarne le radice: el maggiore nasce volentieri acanto. le selve, et le mache (sic), che siano fresche et ombrose: fa le radice piccole, che vanno per traverso la terra, negre et legnose: ma quella che amazza $\mathrm{i}$ vermi le fa succhiose, et grassette, che non si ponno seccar cusì presto, per tal rispetto le figure del libro vostro ve le potrà insegnare etc.

(Bibl. Comun. di Siena, Cod. D. VII. 8 c. 93). 
Doc. VI.

\section{Molto Mag.co Fr.llo Hon.do}

La vostra delli 6 del presente con il viluppetto ricevei li 18 detto da certi nostri frati tornati dal Capitolo di Perosa et subito mandai a nostra sorella i vostri lavori per camise; il ritratto del S. Matthioli b. m. mi è stato caro, parendomi molto simile al suo ritratto che si vede ne i suoi libri, è ben vero che sono un poco differenti per rispetto dell'habito che quello in stampa è fatto con la beretta e zazzera alla tedesca, et questo con beretta piccola 0 berettino che lo fa somigliare propriamente a un castraporcelli di quelli di Cassia et Norsia: similmente il legno quercino me è stato caro et voglio operarlo per le vertigine che a questi giorni mi travagliano un poco dì dì : anchor che adesso non mi dia fastidio.

Dalla Roccha Q.ta, li 22 maggio 1581.

Vostro Amor.le f.llo

Ghirardo Cibo.

(Bibl. Comun. di Siena. Cod. D. VII, 7, c. 56).

Doc. VII.

Dalla Roccha Q.ta li 25 Maggio 1581.

..... di novo vi ringratio del ritratto del s. ${ }^{r}$ Mathioli b. m. quale ogni giorno mi è più caro, per rispetto della memoria di quell'homo tanto eccellente et dabene, al quale seinpre per le qua lità sue ho portato, et portarò sempre affettione, che in quell'altro secolo potrâ chiarirsene, se sarà in loco di salvatione, per le preghiere che farò fare per l'anima sua, et sacrifitii in altari privilegiati, che qui ne havemo già dui, da poco tempo in qua, et in cambio di questo voglio piacendo a Dio mandarvi una figurina colorita di s.ta Catarina che sarà de mia mano etc.

(Bibl. Comun. di Siena. Cod. D, VII. 4, c. 158).

Doc. VIII.

\section{Dalla Rocca Q.ta li 29 di Agosto 1581.}

.... mi dispiace che il legno quercino mandatomi sia andato per mala via, massime essendo come questo, che ho al presente, et odorifero: mi dubito che quei dui pezzi piccoli, che mi mandasti la prima volta, per non sentirli odor alcuno, et molto bianchi di dentro, siano de melo o de altri arbori domestichi: quanto l'esser bono per le vertigine portandolo, come ben insegna il Matthiolo, venne dalla S.ra Cornelia Varroma di f. m. per cosa eccellente et esperimentandolo io sopra di me da 7 anni sono, che tal infirmità mi cominciò travagliare, et bene, a me pare che assai 
mi giovasse, ancor che lo portasse senza la sua scorza, come non vuol essere, che si bene il legno sarà grossetto si potrà un pochino segato spaccarlo per mezzo, o vero in terzo, o in quarto, che verrà pur haver da una banda la sua scorza, poi pertugiarlo per metterli il filo, et attaccarlo al collo, che tocchi la bocca dello stomaco: et portarlo per molti giorni, et facendolo voi, credo ve ne ritrovarete contento etc.

(Bibl. Comun. di Siena. Cod. D. VII. 8, ‘ 130).

Doc. IX.

Dalla Roccha Q.ta li 26 di Ottobre 1586.

El lapatio minore, cioè romice minore: tengo che sia quella spetie piccola, che vulgarmente da noi è chiamato rapacciolo, che si mangia di primavera, et nell'autunno, et a me piace assai, et mangione spesso al tempo suo: nè conosco romice più piccola di questa, che ordinariamente le sue fronde passano di poco la grandezza di quelle della piantagine: et asai volte sono minore, le quale nel fondo loro si ristringono, et poi si alargano alquanto, quasi come si vede nelle foglie de i naranzi, proprio stanno come questa, che vi ho dipinta con il lapis, acciò meglio la possiate conoscere, et nessuna dell'altre romice hanno il garbo di questa, ma sono seguite fino in fondo etc.

(Bibl. Comun. di Siena. Cod. D. VII. 5, c. 88).

Doc. X.

\section{Molto Mag.co F.llo Hon.do}

... Dispiacendomi del vomito et mal di fianchi al quali vi disegnava ritrovare rimedio acció non vi dia si spesso travaglio et pena et bevere tanta acqua non è bono nè tan poco i vini bruschi nè i dolci perchè opilano: nè manco è bene arischiarsi di prender per bocca ciò che ci si insegna. La imperatoria è bona a tal male come ancora la angelica, ma per essere callide assai non convengono quando ci è febbre. La imperatoria è calda quasi in 4 grado e la angelica in 3 grado, et quel miscuglio di butirro et mel rosato a me non piace, piaceme che sia capitata la mia con la foglia del tasso barbasso che si opra per la quartana.... Ho voluto dirvi questo accio crediate che con l'ordinamento bene ne guarirete. E credo faria molto a proposito di pigliare questo sciroppo cioè: capel venere, del fresco citrae, che è la scolopendria di Dioscoride pur fresco, con un poco di cannella et il tutto far bollire con acqua in un pignatino novo vitriato che tenga un bicchiere e mezzo et farlo tanto bollire che cali un terzo et la matina beverne che sia tepido 
un bicchiere et il siroppo si facci ogni sera per la matina che altramente se putrefaria et quando lo pigliarete ci potrete mettere un pezzo di zuccaro quanto una castagna overo con un poco di gileppe. Si potria ancor fare il sciroppo con il brodo di polastro ehc habia bollito solo il bollore, et schiumato perchè questo è migliore per il stomaco e dà anche sostanza.

Ancora mi venne li 15 del presente l'ultima vostra di 7 detto. Con le molte misure de quadretti et la misura e disegno dell'orto vostro: il quale io non intendo bene che saria meglio misurarlo a piedi che a braccia, et mandarme la misura del piede con uno spaghetto o filo, ch'a braccia non potria risponder giusto, e saria ancor bene da muro a muro anchor che ci sia la pergola, misurar anco il spatio quanto che occupa di quel sito, e se la pergola piglia tutto il spatio da ogni banda; overo la parte che piglia o una, o 2, o 4, ec. et così intenderò meglio, ma non vedo che in sì piccolo spazio si possa far cosa garbata, bisognandovi le stradelle che per poterci andare non ponno essere di manco di un bon piede che è quanto più stretta si pol fare, ogni manco che siano non ci si potrà caminare e qui vi sarà la misura del piede nostro de' muratori: e quando non si usi far le misure di piedi li, ma di palmi, mi manderete la misura del palmo.

Di Roccha q.ta li 25 di aprile 1588.

Mi scordavo dirvi et ringratiarvi della ricetta della quartana che mi è stata carissima pel servitio de molti et io in cambio de questa vi manderó la ricetta contro la pontura che si fa solo con olio comune et acqua di pozzi vivi, adesso non lo tempo di farlo.

Vostro amor.le fr.llo

(Bibl. Comun. di Siena. D. VII. 9. c. 72).

Ghirardo Cibo.

Doc. XI.

Molto Mag.co F.llo Hon.do.

Un prete amico mio mi ha dato una ricetta per il mal di fianchi, che si diletta di secreti e di sperimentarli, e dittomi di haverlo provato con varie persone, sempre con felice successo, et di più che guarisce per sempre. E sarà questo: pigliar comino, anici crudi, menta, maggiorana, mentuccia, salvia, rosmarino e canapione cioè artemisia, le erbe si tritaranno alquanto ed alli semi si darà un'acciaccata e tutte le sudette cose si faranno bollire assieme in bon vino e sapia che tanto si faccia bollire che cavi la sostanza di dette herbe et semi, et poi se ne faccia un servitiale che ho voluto mandarvelo con la speranza n' habbia da giovare e liberarve affatto, non è sì gran cosa un servitiale! 
Anchora per la pontura havuto questo secreto: che un capuccino per rimedio sicurissimo avuto in Genova da un gentilhuomo. Prendesi olio comune che sia bono et dolce once 6 , et once 30 di acqua di pozzo vivo overo di fontana, et mettesi l'olio e l'acqua insieme et si faranno bollire fin tanto che tutta l'acqua sia consumata e il detto olio diase tutto a bere in una volta, che in una sol volta guarirà la pontura, ma l'olio vuole essere caldetto. Io poi per farlo migliore penso sia ben farlo bollire in fiasco a bagno maria. Che si fa in questo modo: si mette il fiasco con l'olio et l'acqua dentro, et si acconcia in un caldaretto con acqua che lo ricopra un poco più di mezzo, che la bocca del fiasco sia aperta et aconciarlo con dui sassi uno per banda aciò che mancando o consumandosi l'acqua non possa andare a galla, et si metterà il fiasco nel caldaro che l'acqua sia fredda, che in questo non potrà crepare che quando si mettesse nell'acqua calda al primo creparia subito. E questa sorte di bollire non lascia pigliare sapore all'ólio nè di fritto nè di fumo. E perchè l'acqua che sta nel caldaro si va consumando per il bollire, bisogna tenere continuamente acqua in una pignatta al foco che sia calda acciò che gittandola nel caldaro non fermi il bollire. E con questo farò fine con raccomandarmivi di core pregandovi sanità. Di Roceha q.ta li 8 di Maggio 1588. La vostra ricetta per la quartana si sperimenterà al presente da un mio caro amico, che l'ha tormentato da 8 mesi et seguita tuttavia.

(Bibl. Comun. di Siena. D. VII. 7. c. 56).

Doc. XII.

De Rocca q.ta $1 .{ }^{\circ}$ Ott. 1592.

M.to Mag.co Fratello H.do.

Ricevei la vostra de' 20 del passato, con la copia della litterina, che m' ha scritto Mons. ${ }^{r}$ Capponi li 29 detto; gia vi ho dato risposta dell (sic) littera, che ricevei dal rimandato indietro dal Padre f. Ventura, con i castellini, et mostre de' vostri alberini, piantati nel vostro giardino, con i loro nomi, con dirvi anco di un' altra herbetta per piantare nel vostro praticello: né mancherò ritrovare semi del litosperno minore, et manderolli, io non so né il tempo, né a che luna bisogni seminarli, non ne havendo mai seminati; ne piantai tre o quattro piante nel giardino che havendo benissimo preso, si seminarono da loro stesse, che in pochi anni prese gran parte del giardino del Vescovato, le semente gli cascano, di ottobre o di novembre, però si pol credere, ch'a tal tempo si possi seminare: come si potrá havere la cognitione della pianta che fa il torna sole, mi sarà caro haverla etc.

(Bibl. Comun. di Siena, D. VII. 4 a c. 110). 





$-$

$-1$ 


\section{Estratto dagli Atti della Società Ligustica di Scienze naturali e geografiche}






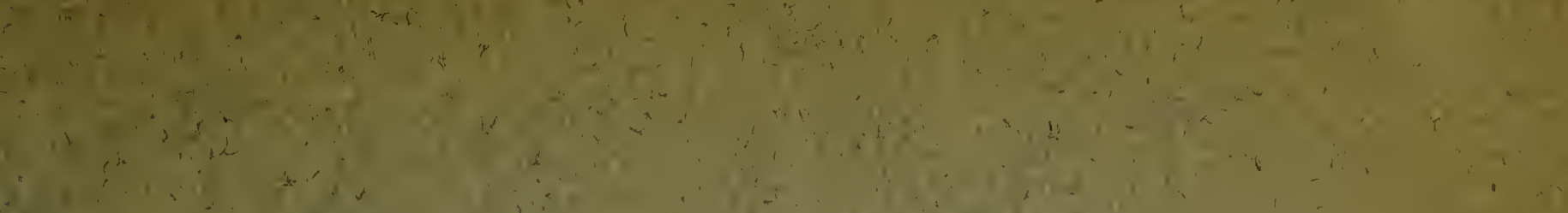

(1) (1)

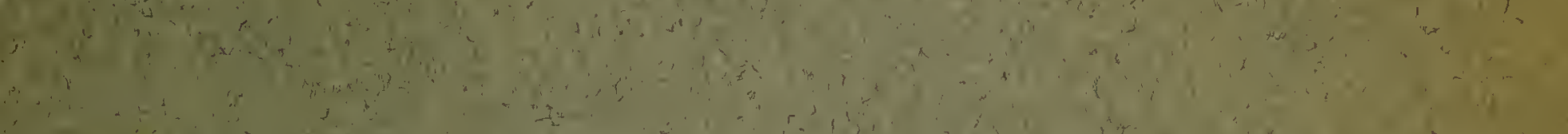

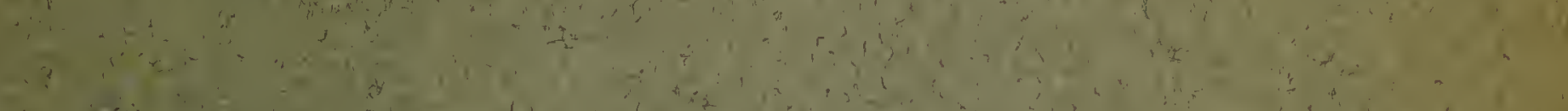
(1)

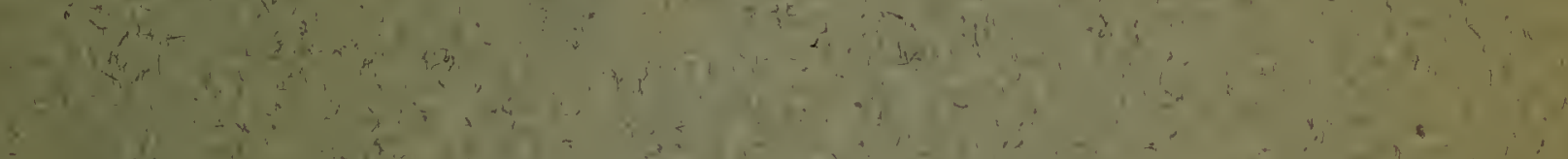

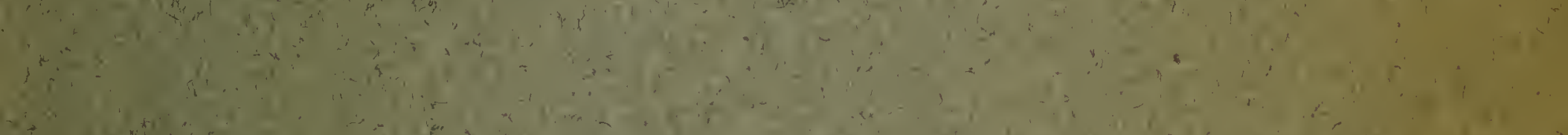
(3)

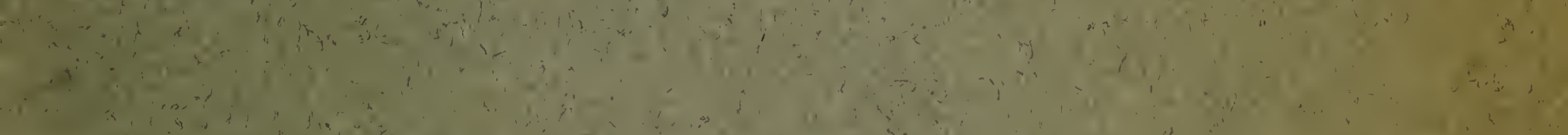

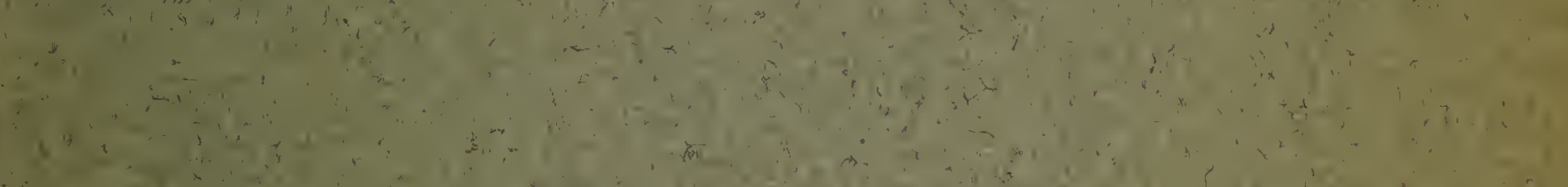

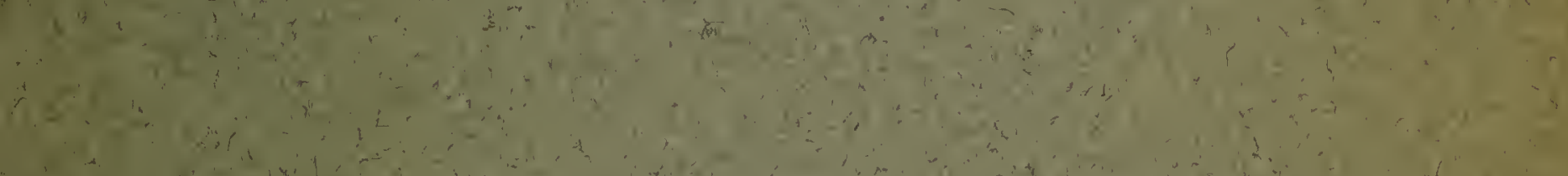

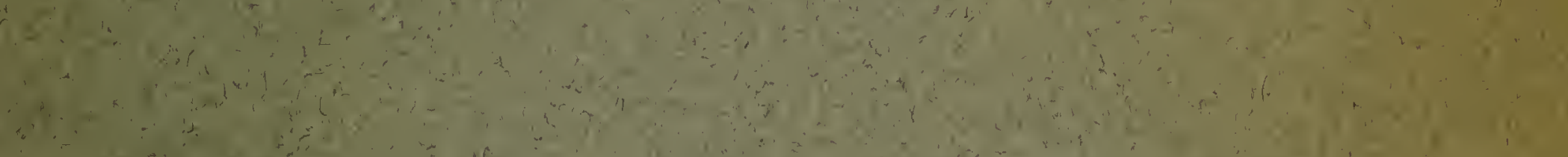

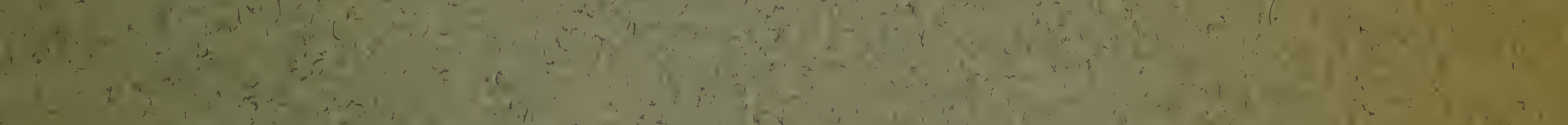

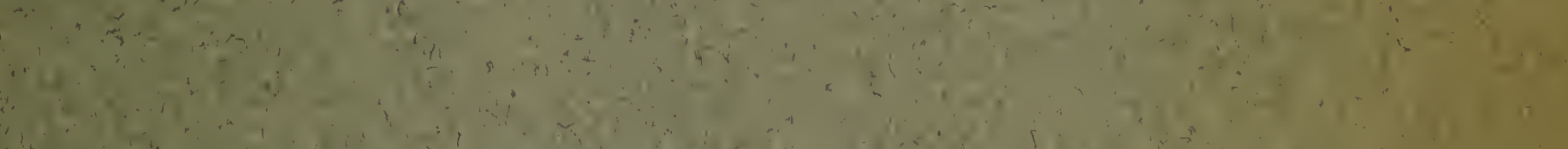
a (3) a $\therefore$

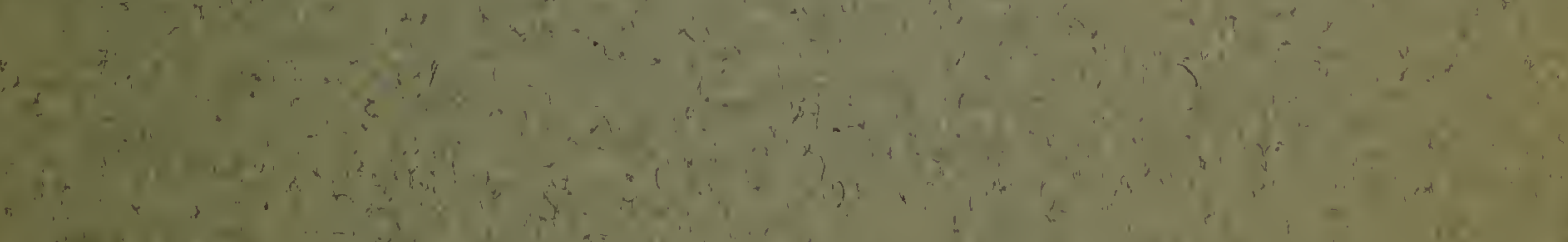
(1)

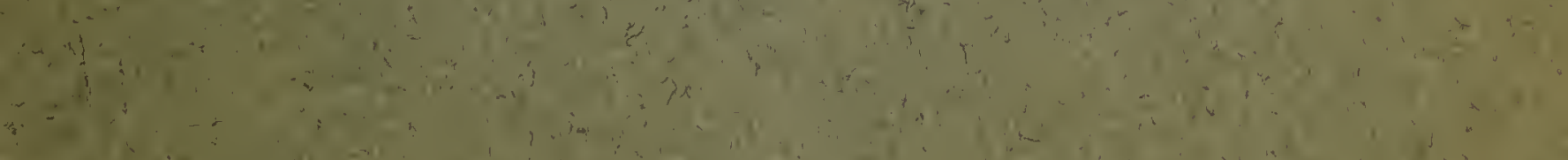

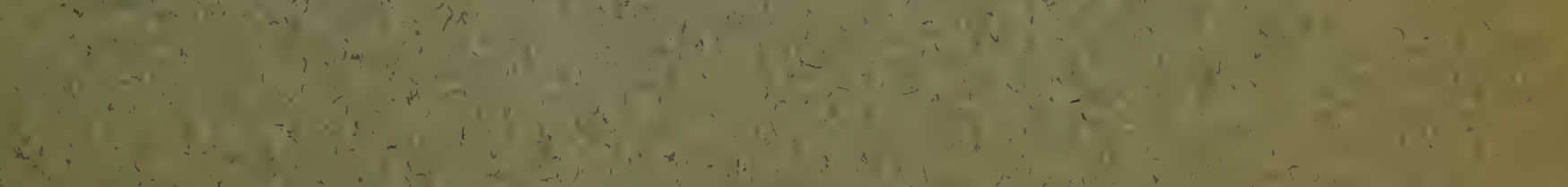
A 


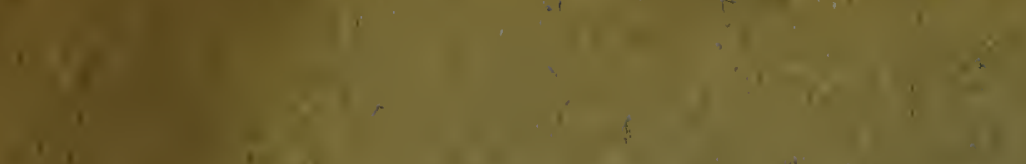

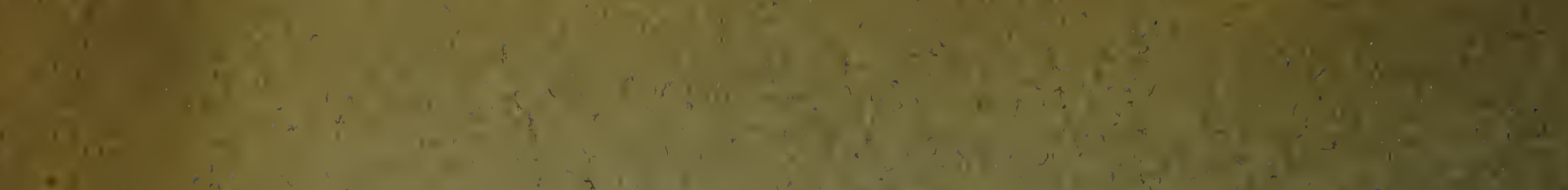



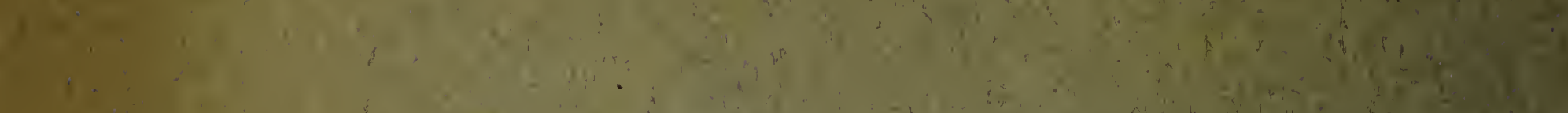
-

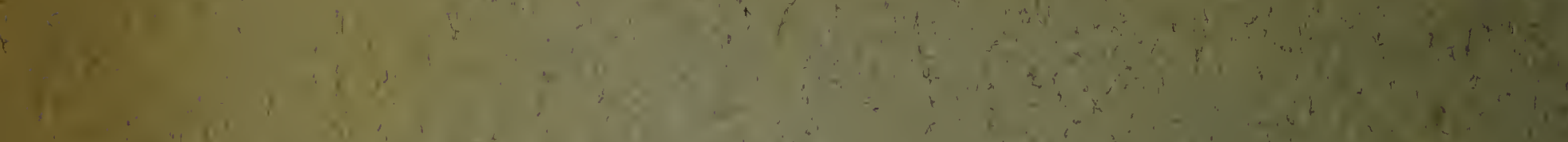

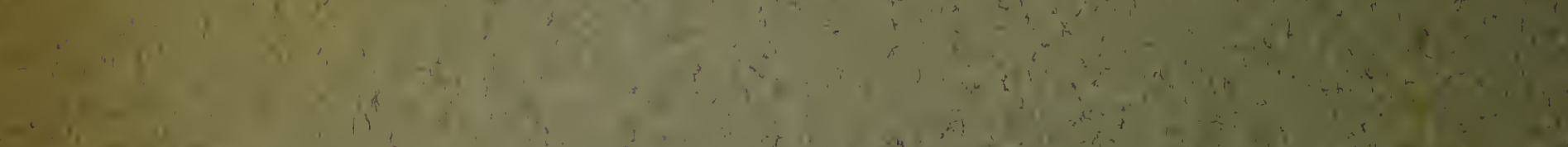
(1)

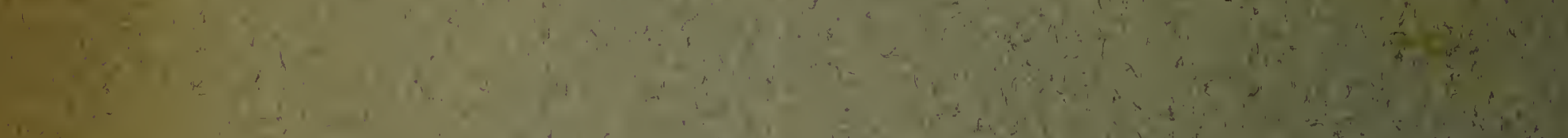

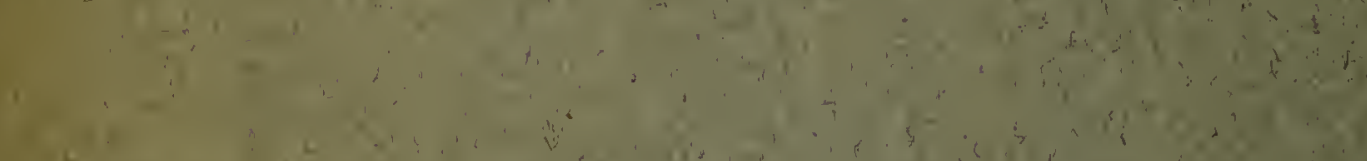

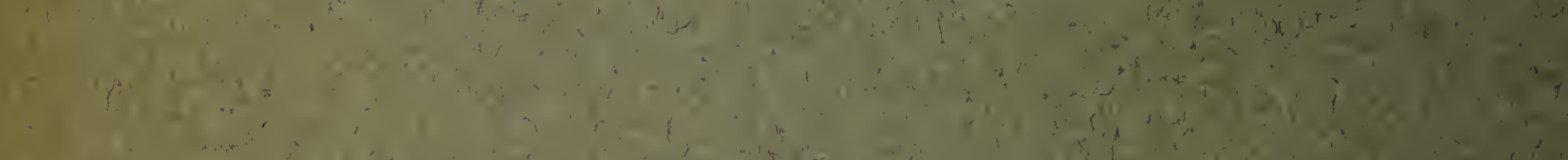

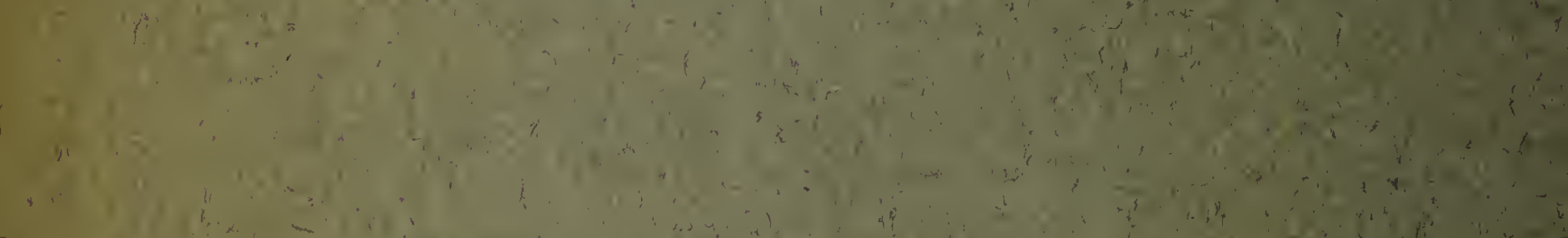

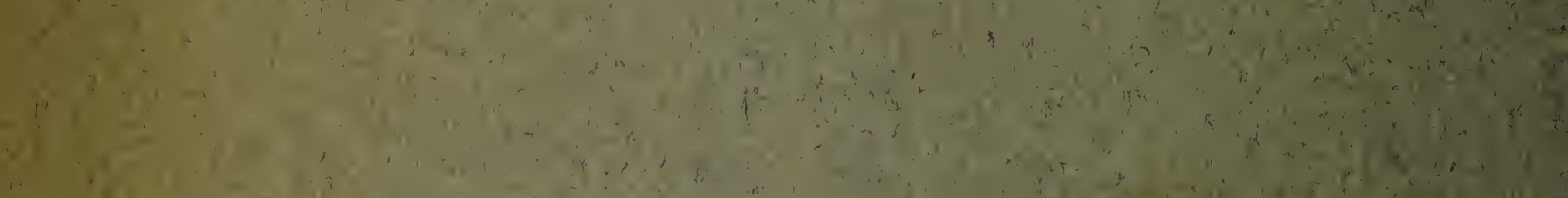

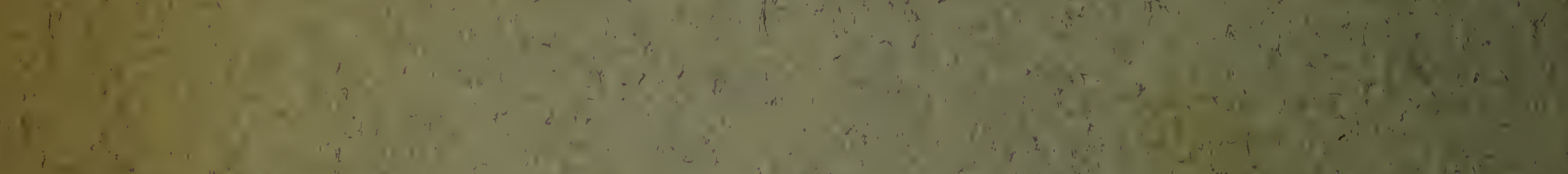

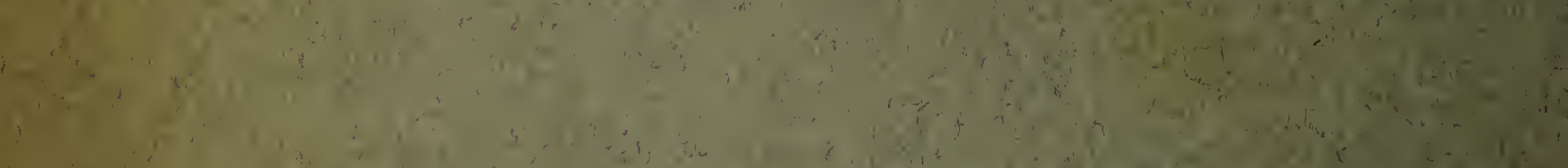

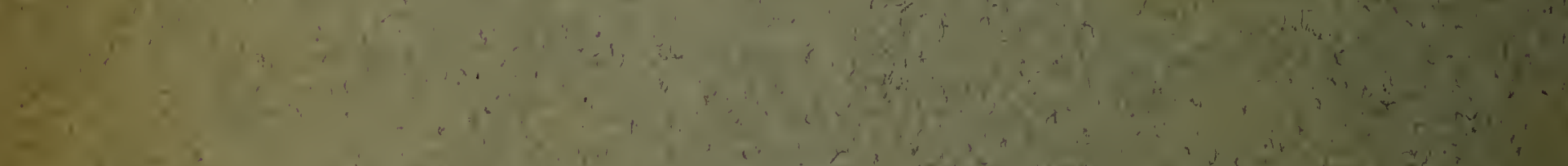

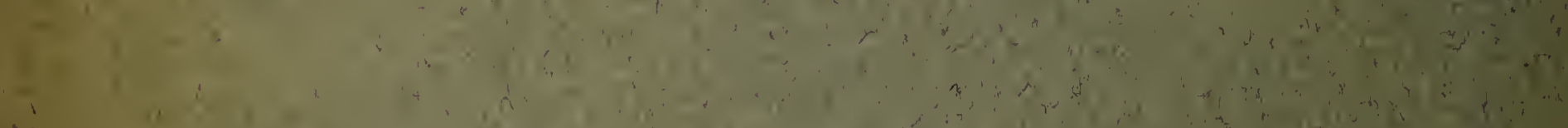

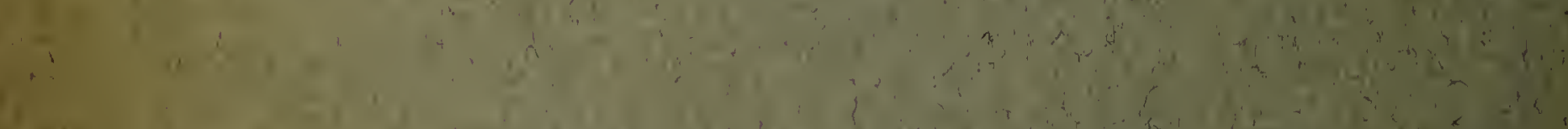

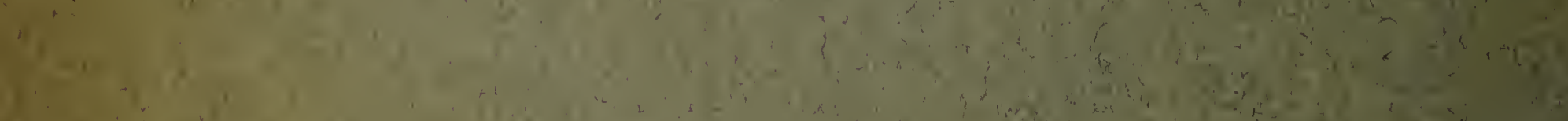

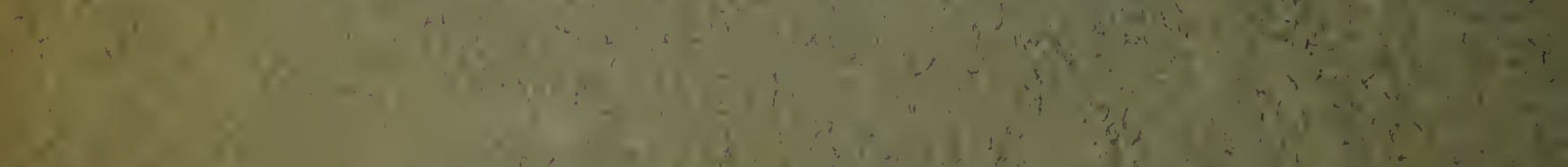

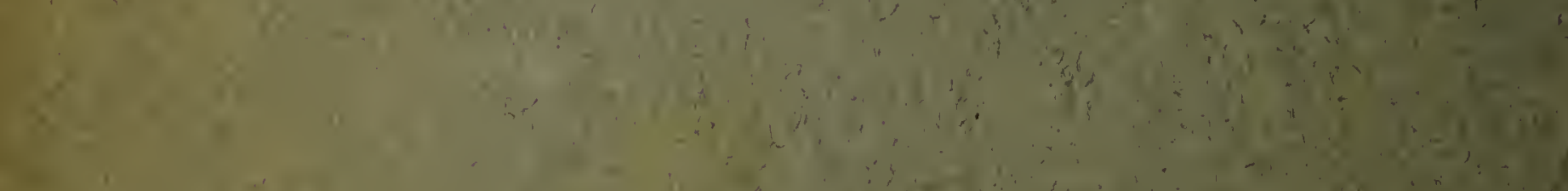
(5)

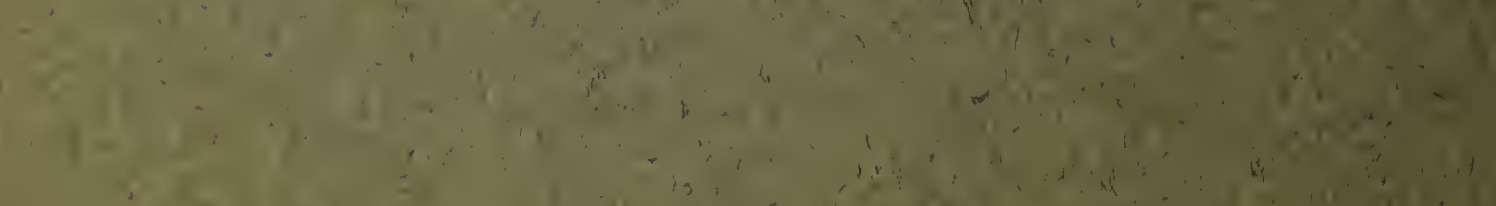

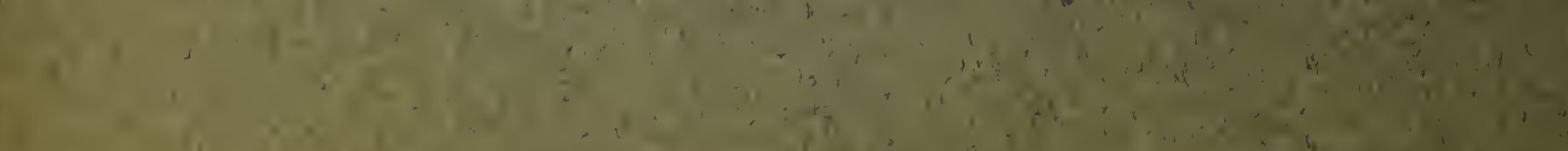

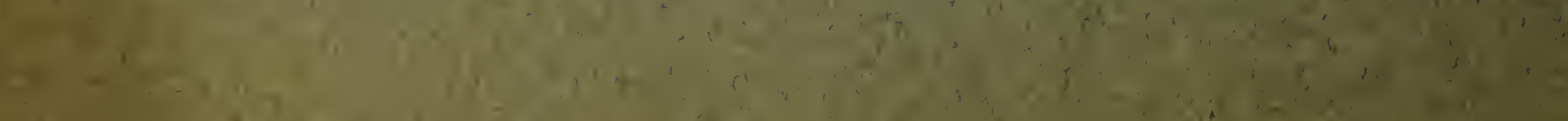

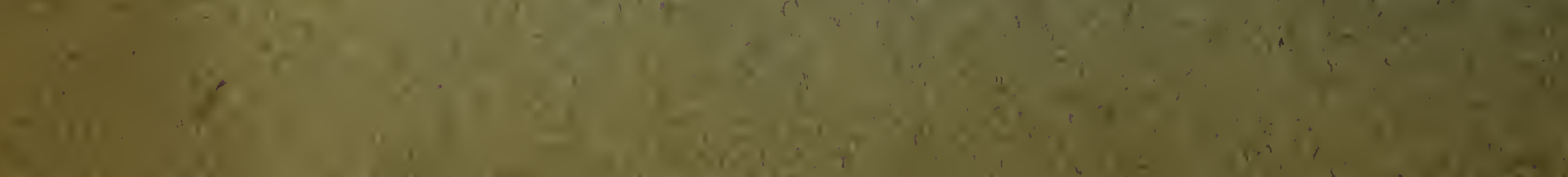

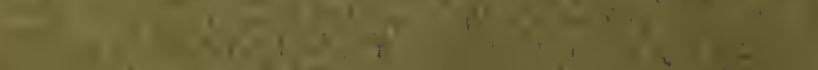

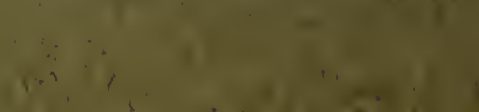

